\title{
Young massive stars in the ISOGAL survey
}

\section{VLA observations of the ISOGAL $l=+45$ field}

\author{
L. Testi ${ }^{1,2}$, M. Felli ${ }^{1}$, and G.B. Taylor ${ }^{3}$ \\ 1 Osservatorio Astrofisico di Arcetri, Largo E. Fermi 5, I-50125 Firenze, Italy \\ 2 Division of Physics, Mathematics and Astronomy, California Institute of Technology, MS 105-24, Pasadena, CA 91125, U.S.A. \\ 3 National Radio Astronomy Observatory, P.O. Box O, Socorro, NM 87801, U.S.A.
}

Received March 22; accepted May 19, 1999

\begin{abstract}
We present VLA radio continuum observations at 3.6 and $6 \mathrm{~cm}$ of a $\sim 0.65$ sq. deg. field in the galactic plane at $l=+45^{\circ}$. These observations are meant to be used in a comparison with ISO observations at 7 and $15 \mu \mathrm{m}$ of the same region. In this paper we compare the radio results with other radio surveys and with the IRASPSC.
\end{abstract}

At 3.6 and/or $6 \mathrm{~cm}$ we detect a total of 34 discrete sources, 13 of which are found in five separate extended complexes. These are all multiple or single extended thermal ultra-compact HII (UCHII) regions. While for each of these complexes an IRAS counterpart could be reliably found, no IRAS counterpart could be reliably identified for any of the remaining 21 sources. Of these 21 compact sources, six are candidate UCHII regions, and the other 15 are most probably background extragalactic non-thermal sources.

The five IRAS sources associated with the radio continuum complexes all satisfy the Wood \& Churchwell (1989a; WC89) color criteria for UCHII. None of the other 38 IRAS point sources present in our surveyed field show the same colors. This fraction of WC89 type to total IRAS sources is consistent with what is found over the entire galactic plane. The fact that, when observed with a compact VLA configuration, the IRAS sources with "UCHII colors" are found to be associated with arcminute-scale extended sources, rather than with compact or unresolved radio sources, may have important implications on the estimated lifetime of UCHII regions.

Key words: HII regions - galaxy: stellar content — stars: early-type — stars: formation — radio continuum: ISM

Send offprint requests to: Testi: Arcetri, lt@arcetri.astro.it

\section{Introduction}

The present work is part of a larger project to study the galactic plane (ISOGAL, Omont \& Blommaert 1997; Pérault et al. 1996). During the ISO mission, the ISOGAL consortium observed with ISOCAM at 15 and $7 \mu \mathrm{m}$ selected parts of the galactic plane (about 18 sq. deg. distributed along the inner galactic disk) in order to study the stellar populations in the inner galaxy, with a sensitivity and resolution two orders of magnitude better than IRAS. The main scientific goal of the ISOGAL project was the study of the distribution and properties of the AGB stars. However, the survey is unbiased, with the only exception of excluding from the surveyed area strong IRAS sources (with $12 \mu \mathrm{m}$ flux densities greater than 6-10 Jy) in order to avoid saturation effects. Thus the survey data can be used to study any other type of mid-IR source present in the galactic plane, as for instance the less numerous HII regions associated to young massive stars.

For a proper identification of source types, the ISOGAL results need to be compared with observations at other wavelengths. In particular, for the study of AGB stars comparisons with near IR observations, taken primarily with DENIS (Epchtein 1998), are useful. For the study of HII regions comparisons with radio continuum surveys are more appropriate.

A large fraction of the northern sky galactic fields covered by ISOGAL have already been observed at $6 \mathrm{~cm}$ (5 GHz) with the VLA (see Becker et al. 1994 and references therein), and a comparison of the two surveys is underway. However, these radio observations terminate at $l=+40^{\circ}$ and there were no high frequency (e.g. $\geq 5 \mathrm{GHz}$ ) radio continuum observations for the ISOGAL field at $l=+45^{\circ}$. Observations at lower frequencies, such as the 1.4 GHz NRAO-VLA Sky Survey (NVSS - Condon et al. 1998), are inadequate to detect the younger and more dense compact HII regions, which may be optically thick at $1.4 \mathrm{GHz}$. 
Given our interest, within the ISOGAL team, to study the young massive stars, we decided to observe the $l=$ $+45^{\circ}$ field at high frequencies with the VLA, to provide a data base comparable to that of Becker et al. (1994). In order to obtain radio spectral index information we covered at 6 and $3.6 \mathrm{~cm}$ an area slightly larger than the $l=+45^{\circ}$ ISOGAL field.

The selection of the ISOGAL galactic plane fields does not follow any ad hoc criterion, but is based on symmetrically spaced samples on both sides of the Galactic Center, with the spacing increasing with distance from the Galactic Center. The $l=+45^{\circ}$ field happens to be located tangent to a spiral arm of our Galaxy, the Scutum arm (see e.g. Kurtz et al. 1994). Inspection of the $4.875 \mathrm{GHz}$ galactic plane survey of Altenhoff et al. (1978) shows that there is very weak diffuse galactic background emission in this direction. Only 7 sources of the Altenhoff et al. catalogue fall in our surveyed area or at its borders (see Table 4$)$. One of these $(44.786-0.490)$ is partly outside our surveyed area. Most of these sources are associated with bright IRAS point sources and have not been covered by the ISOCAM observations except for $45.202-0.441$ and $45.341-0.370$.

In this work we present the radio observations and discuss the comparison with other radio surveys and with IRAS data. Comparison with ISOGAL data, as well as with dedicated $J, H, K$ observations of the same field taken with TIRGO will be the subject of following works.

\section{Observations and data reduction}

The ISOGAL field centered at $l=+45^{\circ}, b=0^{\circ}$ was observed at $6(4.9 \mathrm{GHz})$ and $3.6 \mathrm{~cm}(8.5 \mathrm{GHz})$ using the $\mathrm{NRAO}^{1}$ Very Large Array (VLA) in the $\mathrm{C}$ configuration on 1997 August 5 (8 hours). At $6 \mathrm{~cm}$ the observational setup was similar to that used by Becker et al. (1994), the only differences being that our pointing centers are more closely packed and, due to the peculiar geometry of the sub-fields observed with ISO, we covered the field by scanning strips at constant galactic longitude, which required a total of 31 pointings; our integration time per position was $300 \mathrm{~s}$. At $3.6 \mathrm{~cm}$ we used a similar pointing scheme but scaled due to the smaller primary beam. The observing time per position was reduced to $210 \mathrm{~s}$, and the entire field was mapped with 74 pointings. 8 pointings were observed at $3.6 \mathrm{~cm}$ during a 1 hour test run on 1997 July 4, however, due to a bug in the schedule, only some of the pointings fell in our survey region. For the sake of completeness we will also report the results for the 3 pointings outside our formal survey region that form a spur in position angle 30 degrees.

\footnotetext{
1 The National Radio Astronomy Observatory is a facility of the National Science Foundation operated under agreement by the Associated Universities, Inc.
}

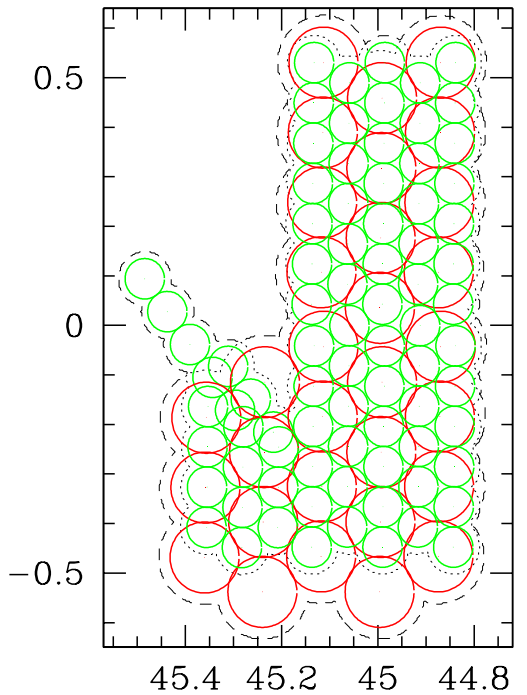

Fig. 1. At each pointing position a circle with diameter equal to the VLA primary beam FWHM is shown. Grey circles represent $3.6 \mathrm{~cm}$ pointings, black circles $6 \mathrm{~cm}$ pointings. The dotted line marks the boundaries of the area observed at both frequencies, the dashed line encompasses the area observed at either of the two bands. In both cases we considered only the area where the a primary beam attenuation is less than a factor of 3. Axes are galactic longitude and latitude (degrees)

Due to the ill-determined primary beam correction and the rapid loss of sensitivity far from the pointing center, we searched for sources only the area where the primary beam attenuation is less than a factor of 3 . With this constraint, we covered an area of $\sim 0.620 \mathrm{sq}$. deg. at $6 \mathrm{~cm}$, and $\sim 0.525$ sq. deg. at $3.6 \mathrm{~cm}$. In Fig. 1 we show all the pointing positions: the small grey circles represent the VLA primary beam HPBW at $3.6 \mathrm{~cm}\left(4.9^{\prime}\right)$, while the larger black circles those at $6 \mathrm{~cm}\left(8.6^{\prime}\right)$. The dotted line show the boundaries of the area covered at both wavelengths $(\sim 0.493$ sq. deg.), the ISOGAL sub-fields are included in this area, the dashed lines mark the boundary of the field observed either at 6 and/or $3.6 \mathrm{~cm}(\sim 0.652 \mathrm{sq}$. deg.).

Frequent observations of the quasar $1922+155$ were used for gain and phase calibration, while the flux density scale was determined by observations of $3 \mathrm{C} 286$. The calibration is expected to be accurate within $10 \%$. We imaged all the fields using the AIPS IMAGR task with natural weighting, the resulting synthesized beam varied somewhat from field to field depending on the hour angle at which each field was observed, typical FWHM values are $\sim 6^{\prime \prime}$ at $6 \mathrm{~cm}$ and $\sim 3^{\prime \prime}$ at $3.6 \mathrm{~cm}$.

\subsection{Sensitivity}

Due to the VLA primary beam attenuation and the different noise values in the various fields, the sensitivity of our observations is not expected to be uniform accross the observed region. Using our knowledge of the VLA 

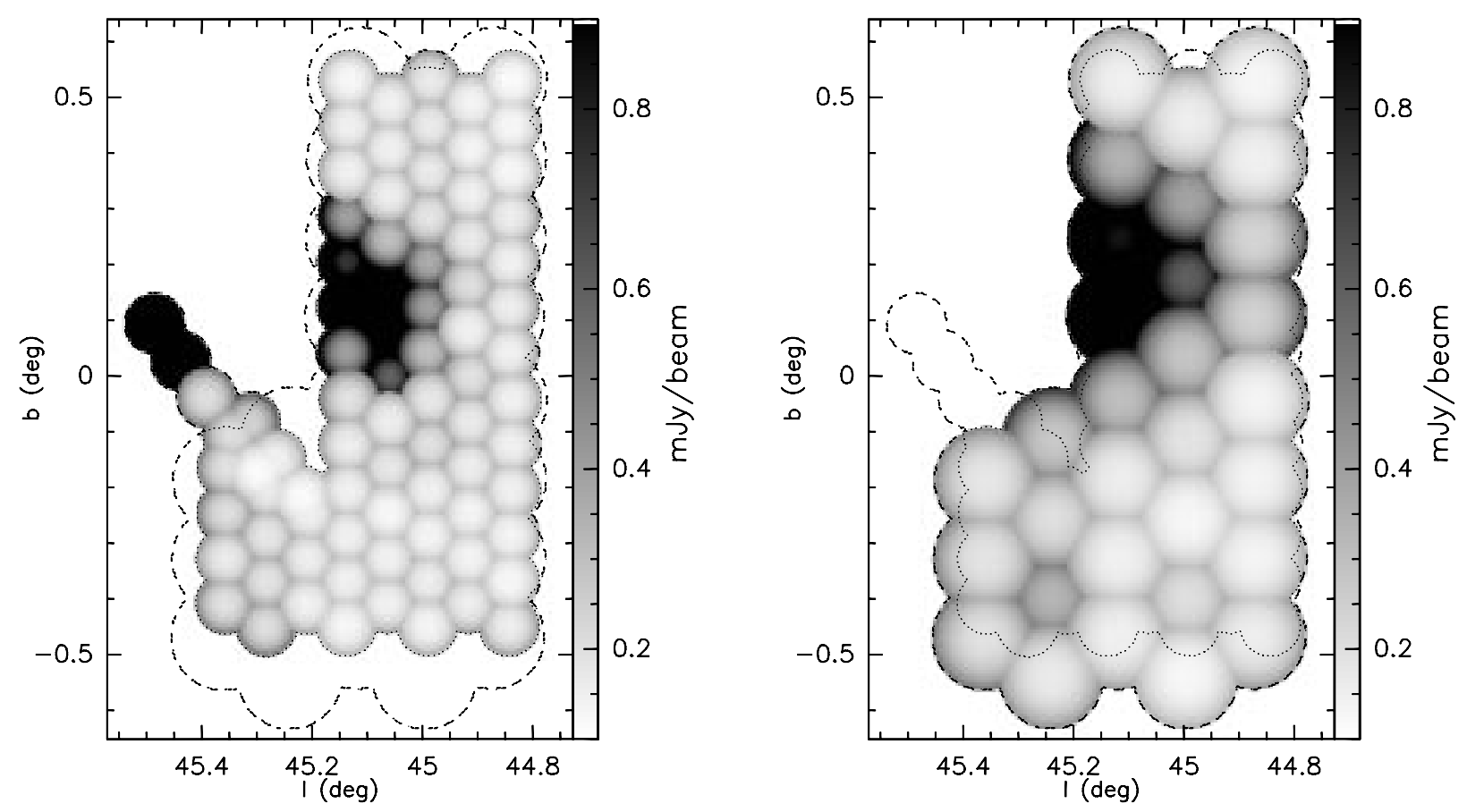

Fig. 2. Computed noise maps for the 3.6 and $6 \mathrm{~cm}$ observations (left and right, respectively). Dotted and dashed lines as in Fig. 1. The on-axis noise level in the black areas can be as high as $8 \mathrm{mJy} /$ beam

primary beam attenuation pattern and the measured onaxis rms level in each of the observed fields, we computed the sensitivity maps for our survey at 3.6 and $6 \mathrm{~cm}$ (see also Zoonematkermani et al. 1990 and Becker et al. 1994). The measured on-axis noise level in the maps is generally $\sim 0.12-0.15 \mathrm{mJy} /$ beam at both frequencies, with the exception of some fields close to the bright complexes located at $l \sim 45^{\circ} 10, b \sim 0.13\left(\alpha(2000)=19^{\mathrm{h}} 13^{\mathrm{m}} 27^{\mathrm{s}} \delta(2000)=\right.$ $\left.10^{\circ} 53^{\prime} 35^{\prime \prime}\right)$ and $l \sim 45.45, b \sim 0.06\left(\alpha(2000)=19^{\mathrm{h}} 14^{\mathrm{m}} 21^{\mathrm{s}}\right.$ $\delta(2000)=11^{\circ} 09^{\prime} 13^{\prime \prime}$ ) which have a higher noise level (in the range $1-8 \mathrm{mJy} /$ beam) due to residual phase and amplitude errors.

The computed rms maps are shown in Fig. 2, the area of each pixel $\left(10^{\prime \prime} \times 10^{\prime \prime}\right)$ corresponds to $\sim 3.5$ beams at $6 \mathrm{~cm}$ and $\sim 14$ beams at $3.6 \mathrm{~cm}$. As seen from Fig. 2 most of the area covered by our survey has a rms sensitivity less than $0.3 \mathrm{mJy} /$ beam at both frequencies. In Fig. 3 we show the cumulative distributions of the pixel values in the rms maps, more than $85 \%$ of the surveyed area has an rms value less than $0.5 \mathrm{mJy} / \mathrm{beam}$.

\subsection{Source extraction}

All images were inspected by means of contour plots and greyscale display to find sources. The images were then corrected for primary beam attenuation using the AIPS task PBCOR before source extraction.

The J2000.0 positions, peak and integrated flux densities and the sizes of the detected sources at both

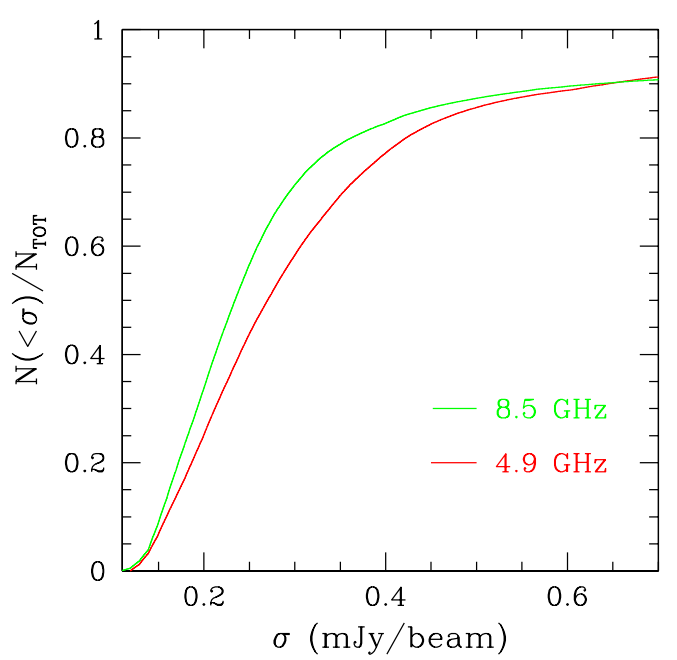

Fig. 3. Cumulative distributions of the noise values in the maps of Fig. 2. The grey line is for $3.6 \mathrm{~cm}$ data, the black line for $6 \mathrm{~cm}$ data

frequencies are listed in Table 1. In general, all the reported detections have a signal-to-noise ratio greater than five in at least one of the two bands. The names assigned to the sources have been derived from their galactic coordinates, as in Becker et al. (1994).

We arbitrarily divided the sources into two categories: 1) compact sources and 2) extended sources. In the first group are all the unresolved sources or the sources with deconvolved sizes of the same order or smaller than the 

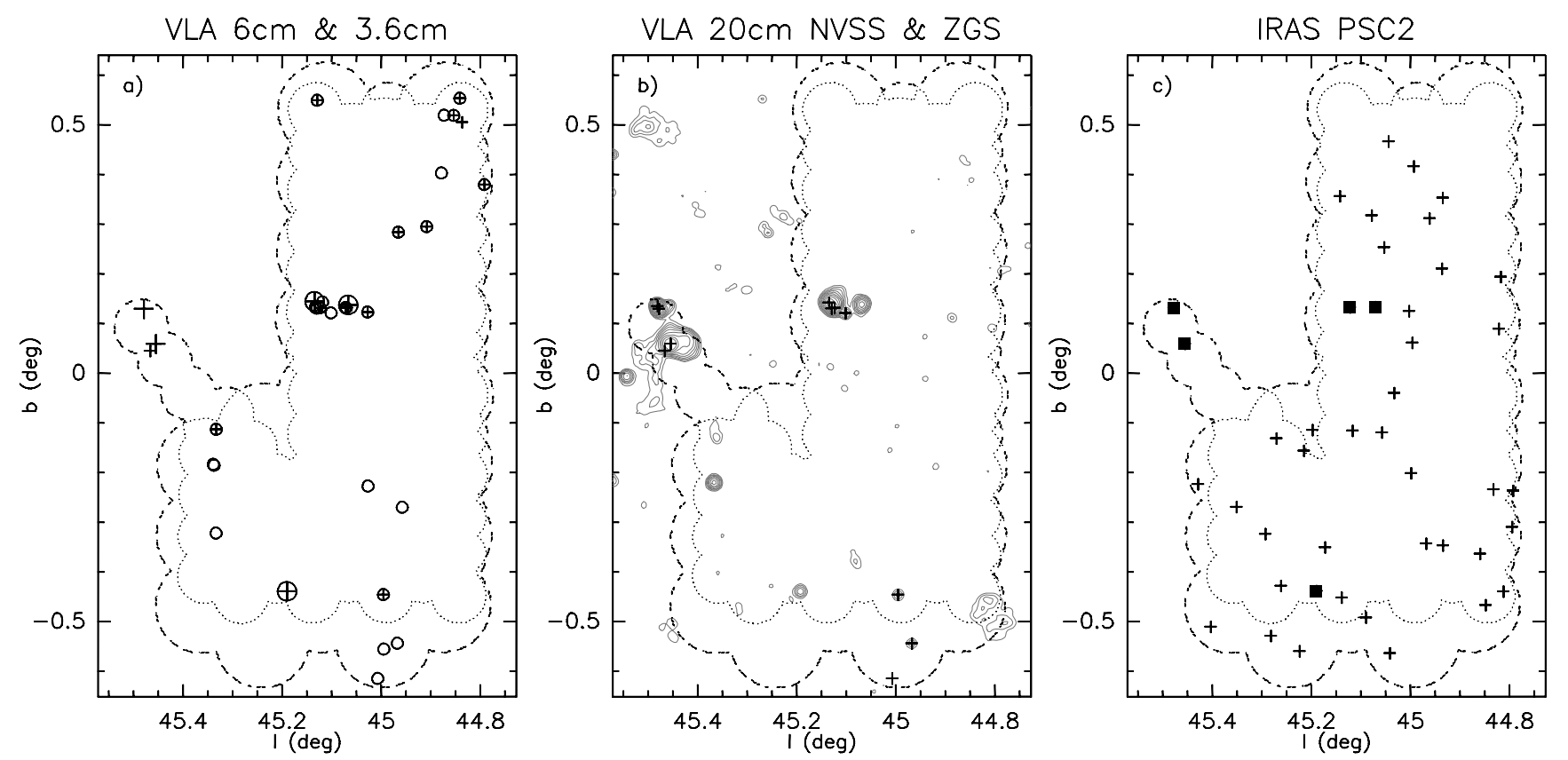

Fig. 4. a) Positions of the detected sources at $3.6 \mathrm{~cm}$ (pluses) and $6 \mathrm{~cm}$ (empty circles), larger symbols represent extended sources; b) VLA 20 cm surveys: sources from Zoonematkermani et al. (1990; ZGS) are shown as pluses, grey contours show the NVSS image of the region; c) the position of the IRAS-PSC2 sources inside our extended survey area are shown as plus symbols, filled squares show the five sources which satisfy the Wood \& Churchwell (1989a) color criteria

synthesized beam FWHM. All the extended sources have sizes much greater than the synthesized FWHM, and thus they may be partially resolved out by the interferometer. The flux densities (and sizes) reported in Table 1 for these sources should be considered as lower limits. We shall see in the following that this arbitrary distinction based on observational considerations reflects also some intrinsic physical difference between the two groups.

At large distances from the pointing center, the correction factor due to the VLA primary beam attenuation may be large, and hence the source flux density could be ill-determined. In Table 1 the source that has the maximum correction factor applied is source \#5, which is close to the edge of the surveyed area and has correction factors $\sim 2.1$ at $6 \mathrm{~cm}$ and $\sim 2.5$ at $3.6 \mathrm{~cm}$. All other sources, with the exception of \#22 and \#29 at $6 \mathrm{~cm}$, have correction factors lower than 2.0.

The positions of all the detected sources within our surveyed region are shown in Fig. 4a, where pluses represent $3.6 \mathrm{~cm}$ sources (21), and circles represent $6 \mathrm{~cm}$ sources (29), and larger symbols represent extended sources. Contour plots for all the detected sources are shown in the Appendix.

\section{Comparison with other surveys}

\subsection{VLA $20 \mathrm{~cm}$ surveys}

The observed field is included in the VLA $20 \mathrm{~cm}$ galactic plane survey (ZGS; Zoonematkermani 1990) and in the NVSS (Condon et al. 1998). Both these surveys used the VLA at $20 \mathrm{~cm}(1.4 \mathrm{GHz})$, however, the ZGS used the moderately extended B array and has a typical peak flux density sensitivity of $25 \mathrm{mJy} /$ beam and a synthesized beam of $\sim 5^{\prime \prime}$ (FWHM), while the NVSS used the most compact $\mathrm{D}$ array with a flux density limit of $\sim 2.5 \mathrm{mJy} /$ beam $(\sim 0.5 \mathrm{mJy} /$ beam $\mathrm{rms})$ and an angular resolution of $\sim 45^{\prime \prime}$.

Given the relatively low sensitivity, and the similar $(u, v)$ sampling with our $6 \mathrm{~cm}$ observations, we expect to detect all the ZGS sources in our maps (see also Becker et al. 1994). On the other hand, due to the much higher sensitivity of the NVSS and its ability to detect extended structures, many of the fainter $20 \mathrm{~cm}$ sources with nonthermal spectral indexes and/or sizes greater than $10^{\prime \prime}$ will not be detectable in our observations. In Fig. 4b we show the positions of all the ZGS (11 - pluses) overlaid on the contour plot of the NVSS image of our survey region.

In Table 2 the results of the correlation between our catalogue and the $20 \mathrm{~cm}$ surveys is presented. The relevant parameters (names, positions, flux densities and sizes) of the $20 \mathrm{~cm}$ sources are from the published catalogues (Zoonematkermani et al. 1990 for the ZGS and the deconvolved data from the fits catalogue available on the World Wide Web at http://www.nrao.edu in October 1998 for the NVSS). The matching criterion used is positional coincidence: ZGS sources are considered to be associated with our sources if the positional difference is less than half a beamwidth for point sources, or if the source position falls within the boundary of one of our extended sources; 
Table 1. Detected radio sources

\begin{tabular}{|c|c|c|c|c|c|c|c|c|c|c|}
\hline \multirow[b]{2}{*}{$\#$} & \multirow[b]{2}{*}{ Name $^{a}$} & \multirow[b]{2}{*}{$\begin{array}{c}\alpha \\
(2000)\end{array}$} & \multirow[b]{2}{*}{$\begin{array}{c}\delta \\
(2000)\end{array}$} & & & \multirow[b]{2}{*}{$\begin{array}{l}\text { Shown in } \\
\text { Figure }^{c}\end{array}$} \\
\hline & & & & $\begin{array}{c}F_{\mathrm{p}}^{b} \\
(\mathrm{mJy} / \text { beam })\end{array}$ & $\begin{array}{c}F_{\mathrm{i}}^{b} \\
(\mathrm{mJy})\end{array}$ & $\begin{array}{l}d^{b} \\
\left(^{\prime \prime}\right)\end{array}$ & $\begin{array}{c}F_{\mathrm{p}}^{b} \\
(\mathrm{mJy} / \text { beam })\end{array}$ & $\begin{array}{c}F_{\mathrm{i}}^{b} \\
(\mathrm{mJy})\end{array}$ & $\begin{array}{l}d^{b} \\
\left({ }^{\prime \prime}\right)\end{array}$ & \\
\hline 1 & G044.841+0.554 & $19: 11: 24.65$ & $+10: 50: 20.7$ & $1.4 \pm 0.2$ & 1.6 & 0 & $0.8 \pm 0.2$ & 1.1 & 1 & A1 \\
\hline 2 & G044.854+0.519 & 19:11:33.51 & $\begin{array}{l}+10: 50: 02.8 \\
\end{array}$ & $0.7 \pm 0.2$ & 0.7 & 1 & $0.6 \pm 0.2$ & 1.6 & 3 & A1 \\
\hline 3 & G044.837+0.506 & $19: 11: 34.52$ & $+10: 48: 46.0$ & $<0.3$ & - & - & $0.7 \pm 0.2$ & 0.7 & 0 & A2 \\
\hline 4 & G044.873+0.520 & $19: 11: 35.64$ & $\begin{array}{l}+10: 51: 05.3 \\
\end{array}$ & $0.9 \pm 0.2$ & 1.4 & 5 & $<0.6$ & - & - & A2 \\
\hline 5 & G044.792+0.380 & $19: 11: 56.70$ & $+10: 42: 53.5$ & $2.5 \pm 0.2$ & 3.2 & 0 & $1.7 \pm 0.2$ & 2.1 & 0 & $\mathrm{~A} 1$ \\
\hline 6 & G045.129+0.550 & $19: 11: 58.07$ & $\begin{array}{l}\text { +11:05:32.9 } \\
\text { - }\end{array}$ & $0.8 \pm 0.2$ & 1.2 & 0 & $0.5 \pm 0.2$ & 0.5 & 0 & A1 \\
\hline 7 & G044.878+0.403 & 19:12:01.41 & $\begin{array}{l}+10: 48: 09.0 \\
\end{array}$ & $0.6 \pm 0.2$ & 0.9 & 4 & $<0.6$ & ${ }_{-}^{0}$ & - & $\mathrm{A} 2$ \\
\hline 8 & G044.908+0.295 & $19: 12: 28.20$ & $+10: 46: 43.2$ & $1.5 \pm 0.4$ & 1.6 & 1 & $2.0 \pm 0.3$ & 2.5 & 1 & $\mathrm{~A} 1$ \\
\hline 9 & G044.965+0.284 & $19: 12: 37.07$ & $+10: 49: 27.1$ & $18.4 \pm 0.6$ & 20.1 & 1 & $18.5 \pm 0.4$ & 18.1 & 0 & A1 \\
\hline 10 & G045.027+0.123 & $19: 13: 18.94$ & $+10: 48: 16.5$ & $9.0 \pm 0.8$ & 10.1 & 0 & $7.2 \pm 1.1$ & 6.6 & 0 & A1 \\
\hline 11 & G045.070+0.132 & $19: 13: 21.87$ & $+10: 50: 49.0$ & $63.2 \pm 3.4$ & $270^{d}$ & 6 & $57.6 \pm 3.7$ & 106.6 & 2 & A3 \\
\hline 12 & G045.072+0.132 & $19: 13: 22.08$ & $+10: 50: 53.2$ & $128.7 \pm 3.6$ & $270^{d}$ & 0 & $307.3 \pm 3.9$ & 326.1 & 0 & A3 \\
\hline 13 & $\mathrm{G} 045.118+0.143$ & $19: 13: 24.90$ & $+10: 53: 41.1$ & $24.0 \pm 3.6$ & 172.0 & 6 & $<24.0$ & - & - & A4 \\
\hline 14 & $\mathrm{G} 045.101+0.122$ & $19: 13: 27.67$ & $\begin{array}{l}+10: 52: 09.6 \\
\end{array}$ & $16.9 \pm 3.4$ & 34.0 & 5 & $<24.0$ & - & - & A2 \\
\hline 15 & G045.123+0.132 & $19: 13: 27.91$ & $\begin{array}{l}+10: 53: 36.3 \\
\end{array}$ & $1436.1 \pm 3.4$ & 2905.8 & 5 & $1431.6 \pm 17.2$ & 3294.7 & 3 & $\mathrm{~A} 4$ \\
\hline 16 & G045.133+0.133 & $19: 13: 28.81$ & $+10: 54: 09.8$ & $24.0 \pm 3.6$ & 88.0 & 4 & $<24.0$ & - & - & A4 \\
\hline 17 & G045.130+0.131 & $19: 13: 28.83$ & $+10: 53: 56.1$ & $37.2 \pm 3.6$ & 91.0 & 4 & $38 \pm 8.0$ & 77 & 3 & A4 \\
\hline $18^{e}$ & G045.455+0.060 & $19: 14: 21.29$ & $+11: 09: 12.3$ & $-f$ & $\_f$ & ${ }_{-} f$ & $195.0 \pm 4.2$ & $1050.0^{e}$ & 6 & A5 \\
\hline 19 & G045.466+0.045 & $19: 14: 25.66$ & $+11: 09: 26.1$ & $\_f$ & $-_{f}$ & $-f$ & $87.2 \pm 4.6$ & 105.4 & 1 & A2 \\
\hline 20 & G045.026-0.227 & $19: 14: 34.64$ & $+10: 38: 28.7$ & $0.9 \pm 0.2$ & 0.8 & 0 & $<0.6$ & - & - & A2 \\
\hline 21 & G044.958-0.270 & $19: 14: 36.02$ & $+10: 33: 37.7$ & $1.0 \pm 0.2$ & 1.2 & 0 & $<0.6$ & - & - & A2 \\
\hline 22 & G045.333-0.113 & $19: 14: 44.71$ & $+10: 57: 56.7$ & $4.1 \pm 0.5$ & 4.4 & 0 & $3.6 \pm 0.4$ & 4.3 & 1 & A1 \\
\hline 23 & G045.339-0.183 & $19: 15: 00.62$ & $+10: 56: 17.6$ & $2.1 \pm 0.5$ & 3.3 & 3 & $<0.9$ & - & - & A2 \\
\hline 24 & G045.337-0.185 & $19: 15: 00.87$ & $\begin{array}{l}+10: 56: 09.7 \\
\end{array}$ & $1.3 \pm 0.5$ & 1.4 & 0 & $<0.9$ & - & - & A2 \\
\hline 25 & G044.996-0.446 & $19: 15: 18.35$ & $+10: 30: 44.3$ & $7.5 \pm 0.1$ & 7.8 & 0 & $4.2 \pm 0.3$ & 4.6 & 0 & $\mathrm{~A} 1$ \\
\hline 26 & G045.333-0.322 & $19: 15: 29.98$ & $+10: 52: 08.0$ & $1.3 \pm 0.3$ & 2.3 & 3 & $<0.6$ & - & - & A2 \\
\hline 27 & G044.967-0.544 & $19: 15: 36.29$ & $+10: 26: 30.6$ & $6.5 \pm 0.2$ & 6.9 & 1 & $f$ & ${ }_{-} f$ & $\_f$ & A2 \\
\hline 28 & G044.995-0.555 & $19: 15: 41.95$ & $+10: 27: 38.0$ & $1.1 \pm 0.2$ & 1.0 & 0 & $-f$ & $-f$ & $-f$ & $\mathrm{~A} 2$ \\
\hline 29 & G045.007-0.614 & $19: 15: 56.12$ & $+10: 26: 38.1$ & $3.4 \pm 0.2$ & 2.8 & 0 & $\_f$ & ${ }_{-} f$ & ${ }_{-} f$ & A2 \\
\hline \multicolumn{11}{|c|}{ Extended sources } \\
\hline 30 & G045.066+0.138 & $19: 13: 20.5$ & $+10: 50: 50$ & $39.5 \pm 3.0$ & 348.6 & 26 & $14.9 \pm 2.0$ & 433.0 & 26 & A3 \\
\hline 31 & G045.134+0.145 & $19: 13: 26.5$ & $+10: 54: 20$ & $73.0 \pm 3.0$ & 1960.0 & 48 & $60.2 \pm 8.0$ & 1727.7 & 48 & $\mathrm{~A} 4$ \\
\hline 32 & G045.479+0.130 & $19: 14: 08.8$ & $+11: 12: 28$ & $-f$ & $-f$ & $-f$ & $37.2 \pm 2.0$ & 1500.0 & 30 & A5 \\
\hline $33^{e}$ & G045.455+0.059 & $19: 14: 21.3$ & $+11: 09: 10$ & $\_f$ & $\_f$ & $\_f$ & $65.0 \pm 2.0$ & $3450.0^{e}$ & 47 & A5 \\
\hline 34 & G045.190-0.439 & $19: 15: 39.0$ & $+10: 41: 15$ & $7.6 \pm 0.3$ & 95.6 & 36 & $3.5 \pm 0.2$ & 69.7 & 36 & A6 \\
\hline
\end{tabular}

$\left.{ }^{a}\right)$ Derived from galactic coordinates, as in Becker et al. (1994)

$\left.{ }^{b}\right) F_{\mathrm{p}} \equiv$ peak flux density; $F_{\mathrm{i}} \equiv$ integrated flux density; $d \equiv$ size (deconvolved).

$\left.{ }^{c}\right)$ Contour plots for all the detected sources are reported in Appendix.

$\left.{ }^{d}\right)$ Sources \#11 and \#12 are blended together at $6 \mathrm{~cm}$, the separation of the two contribution to the integrated flux is very uncertain, thus we report the integrated flux of both components together.

$\left.{ }^{e}\right)$ Source \#18 is inside the extended source \#33. The integrated flux density of the compact component has been subtacted from the total integrated flux density, the resulting flux has been assigned to source \#33.

${ }^{f}$ ) Not observed.

NVSS sources are considered to be associated if one of our point source falls inside of, or if the boundaries of one of our extended sources overlap with, the deconvolved size of the $20 \mathrm{~cm}$ source. As expected, all the ZGS sources in our surveyed field do have a counterpart at $6 \mathrm{~cm}$. In one case (source \#32 in our list), we considered two ZGS sources as being part of the same (extended) $6 \mathrm{~cm}$ source. In Table 2 , Cols. 1 and 2 report the numbers and names of our sources from Table 1, Cols. 3 to 6 the names, peak and integrated flux densities, and sizes of the ZGS sources, Cols. 7 to 10 the names, integrated flux densities and deconvolved sizes of the NVSS sources, and Col. 11 the IRAS sources names (see Sect. 3.4).

In general, given the higher sensitivity of the NVSS and its ability to detect extended sources that might be resolved out in the ZGS, we expect that all the ZGS sources in our field should be detected in the NVSS as well. The only possible exception is that of very compact high surface brightness sources close or inside large low surface brightness sources with high integrated flux. There are 3 ZGS sources without an NVSS counterpart, one $(045.129+0.131$, associated to our \#17) is indeed inside the bright complex shown in Fig. A4, and thus may be missing from the NVSS catalogue due to confusion. Similarly, the one associated with our \#19 could be undetected in the NVSS due to its proximity to the extended source \#33. Both \#17 and \#19 have thermal spectral indexes (see below and Table 3) and we do not expect them to be variable at $20 \mathrm{~cm}$. On the other hand, the ZGS source associated with \#29 should 


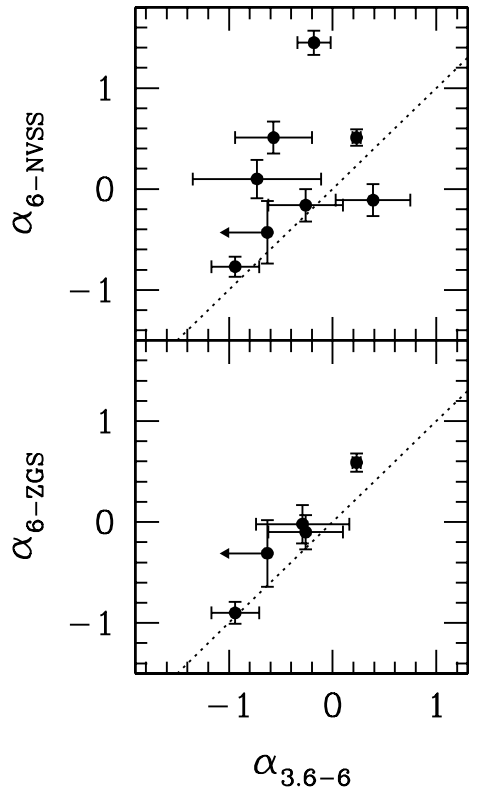

Fig. 5. Comparison between the high and low frequency spectral indexes. Top panel: sources in our survey detected at $20 \mathrm{~cm}$ in the NVSS. Bottom panel: sources detected in the ZGS. In both panels the dotted line represent equal spectral indexes

have been detected in the NVSS, thus for this source, given also its non-thermal spectral index, the only viable explanation for the NVSS non-detection is variability at $20 \mathrm{~cm}$.

Finally, there is a very bright $(\sim 280 \mathrm{mJy})$, unresolved, NVSS source which is undetected in the ZGS and in our survey. This source (clearly visible in Fig. $4 \mathrm{~b}$ at $l \sim 45.35$, $b \sim-0.22$ ) is the high energy source G1915+105 (Mirabel \& Rodríguez 1994). At radio wavelengths is known to be highly variable, with flux densities at $1.4 \mathrm{GHz}$ that can be as high as $\sim 1$ Jy at the peak of radio bursts and below the mJy level during quiescence (Rodríguez \& Mirabel 1999).

In Table 3, Cols. 2 to 6 , we report the radio continuum spectral indexes $\left(\alpha\right.$, defined as $\left.F_{\nu} \sim \nu^{\alpha}\right)$ as calculated from our integrated flux densities and the ZGS and NVSS integrated flux densities. It should be noted that all extended sources are probably partially resolved out in the higher resolution surveys, particularly in our $3.6 \mathrm{~cm}$ images, and thus some of the measured spectral indexes are probably lower limits due to the missing flux at high frequency.

In Fig. 5 we compare the high frequency spectral indexes (those calculated between 3.6 and $6 \mathrm{~cm}$ ) with the low frequency ones (calculated between 6 and $20 \mathrm{~cm}$ ), only the sources inside the area observed both at 3.6 and $6 \mathrm{~cm}$ have been considered. A $10 \%$ error has been assumed for all ZGS and NVSS integrated flux densities (this may be a slight underestimate of the true error for the faintest sources in these surveys). In the upper panel we show the comparison for sources detected in the NVSS and in the lower panel that for sources detected in the ZGS. We find very good agreement between the high frequency and the

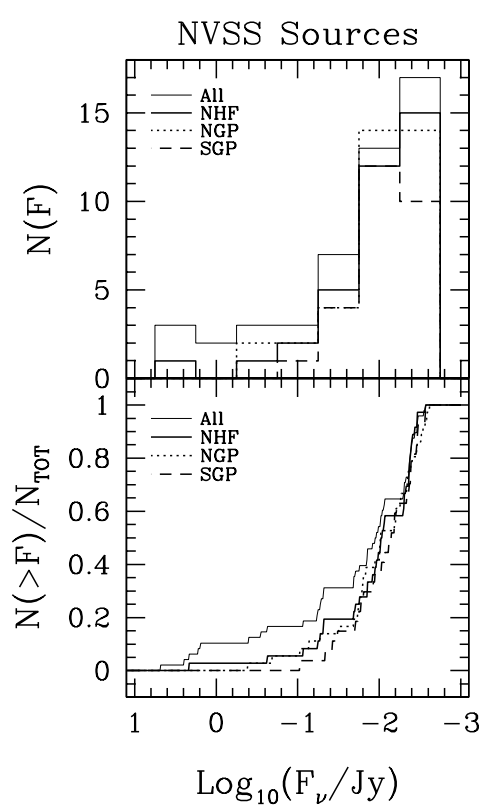

Fig. 6. Top panel: differential luminosity functions of all (All; thin continuous line) and not detected at high frequency (NHF; thick continuous line) NVSS sources inside our field, and of NVSS sources from two $0.652 \mathrm{sq}$. deg. areas close to the northern (NGP; dotted line) and southern (SGP; dashed line) galactic poles. Bottom panel: cumulative luminosity functions for the same sources shown in the upper panel

low frequency spectral indexes for ZGS sources. This is probably due to the matched beams of the observations. In contrast, for NVSS sources, the spread between low and high frequency spectral indexes is much greater. There are two possible explanations for this: 1) the increased sensitivity to extended structures of the NVSS and 2) the greater sensitivity of the NVSS with respect to the ZGS. The increased sensitivity allows for the detection in the NVSS of some thermal sources that are optically thick at low frequency and become optically thin at high frequency (this is probably the case for \#9 and \#34).

\subsubsection{NVSS sources undetected at high frequency}

Most of the NVSS sources in our field (48) are not detected at 6 and/or $3.6 \mathrm{~cm}$. We believe that in most cases the negative spectral index, rather than the different $(u, v)$ coverage between the observations, is the main reason for the non-detection at high frequency. The most plausible explanation is that a large fraction of these NVSS sources are extragalactic objects, with a possible contamination from faint planetary nebulae.

To check whether the $20 \mathrm{~cm}$ flux distribution and source count for the NVSS sources not detected at high frequency are consistent with the population of extragalactic radio sources, we extracted from the NVSS the sources in two areas toward the galactic poles, each of the 
Table 2. Associated ZGS, NVSS and IRAS-PSC2 sources

\begin{tabular}{|c|c|c|c|c|c|c|c|c|c|c|}
\hline \multirow[b]{2}{*}{$\#$} & \multirow[b]{2}{*}{ Name } & \multicolumn{4}{|c|}{ ZGS } & \multicolumn{4}{|c|}{$\mathrm{NVSS}^{a}$} & \multirow{2}{*}{$\begin{array}{l}\text { IRAS } \\
\text { Name }\end{array}$} \\
\hline & & Name & $\begin{array}{c}F_{\mathrm{p}} \\
(\mathrm{mJy} / \mathrm{b})\end{array}$ & $\begin{array}{c}F_{\mathrm{i}} \\
(\mathrm{mJy})\end{array}$ & $\begin{array}{c}d \\
\left({ }^{\prime \prime}\right)\end{array}$ & $\begin{array}{l}\text { Name }^{a} \\
(\text { NVSS J) }\end{array}$ & $\begin{array}{c}F_{\mathrm{i}} \\
(\mathrm{mJy})\end{array}$ & $\begin{array}{l}\text { Size }^{b} \\
\left({ }^{\prime \prime} \times{ }^{\prime \prime}\right)\end{array}$ & $\begin{array}{c}\mathrm{pa}^{b} \\
\left({ }^{\circ}\right) \\
\end{array}$ & \\
\hline 5 & G044.792+0.380 & & & & & $191156+104256$ & 2.8 & $129 \times 55$ & 0 & \\
\hline 9 & G044.965+0.284 & & & & & $191236+104930$ & 3.3 & $58 \times 48$ & 0 & \\
\hline 14 & G045.101+0.122 & $045.101+0.121$ & 41 & 49 & 2.2 & $191327+105217$ & 58.2 & $64 \times 21$ & 22 & \\
\hline 15 & G045.123+0.132 & $045.123+0.132$ & 287 & 1468 & 10.4 & $191327+105338$ & 1540.4 & $21 \times 15$ & 58 & $19111+1048$ \\
\hline 17 & G045.130+0.131 & $045.129+0.131$ & 22 & 93 & 9.1 & & & & & \\
\hline 19 & G045.466+0.045 & $045.466+0.046$ & 16 & 21 & 2.6 & & & & & \\
\hline 25 & G044.996-0.446 & $044.995-0.445$ & 21 & 22 & 0.0 & $191518+103042$ & 20.4 & $22 \times 29$ & 83 & \\
\hline 27 & G044.967-0.544 & $044.967-0.543$ & 21 & 23 & 1.7 & $191536+102630$ & 14.2 & $32 \times 27$ & 0 & \\
\hline 29 & G045.007-0.614 & $045.007-0.614$ & 12 & 10 & 0.0 & & & & & \\
\hline 30 & G045.066+0.138 & & & & & $191320+105054$ & 401.9 & $31 \times 19$ & -83 & $19110+1045$ \\
\hline 31 & G045.134+0.145 & $045.134+0.143$ & 48 & 2245 & 34.8 & $191326+105422$ & 2445.3 & $46 \times 42$ & 53 & \\
\hline \multirow[t]{2}{*}{32} & G045.479+0.130 & $045.477+0.130$ & 97 & 1222 & 17.0 & $191408+111229$ & 1672.6 & $33 \times 14$ & -33 & $19117+1107$ \\
\hline & & $045.480+0.136$ & 62 & 653 & 15.2 & & & & & \\
\hline 33 & G045.455+0.059 & $045.454+0.060$ & 167 & 2207 & 17.5 & $191421+110913$ & 4771.5 & $41 \times 36$ & -20 & $19120+1103$ \\
\hline 34 & G045.190-0.439 & & & & & $191539+104123$ & 50.8 & $20 \times 17$ & -41 & $19132+1035$ \\
\hline
\end{tabular}

$\left.{ }^{a}\right)$ In this table, the "NVSS J" prefixes have been omitted from the names of the NVSS sources.

$\left.{ }^{b}\right)$ Deconvolved major, minor axes and position angle (see Cotton et al. 1998).

two with the same extent of our surveyed region. The number of sources extracted toward the northern and southern galactic poles are 36 and 27 , respectively, these numbers compare relatively well with the 37 NVSS sources without high frequency counterpart in our field. As additional check, in Fig. 6, we show the differential and cumulative luminosity functions for the sources in our field and those in the areas toward the galactic poles. The luminosity function of all the sources in our field (thin line) show an excess of bright sources with respect to the galactic poles, this excess disappears if we plot only the sources without a high frequency counterpart (thick line). This effect is more clear in the cumulative luminosity function plot (Fig. 6, lower panel). More quantitatively, the Kolmogorov-Smirnov test on the cumulative luminosity functions gives a probability lower than $40 \%$ that the NVSS sources in the Galactic poles samples and those in our field are drawn from the same distribution. This probability rises above $80 \%$ if we remove from our sample the sources detected at high frequency and the well known galactic high energy source G1915+105.

\subsection{Effelsberg $5 \mathrm{GHz}$ survey}

As mentioned in Sect. 1, our surveyed region has been covered by the Altenhoff et al. (1978) $5 \mathrm{GHz}(6 \mathrm{~cm})$ single dish survey. The names and peak flux densities of the seven single dish sources inside or partially within our survey boundaries are listed in Table 4 . In the same table, for each source, we report the integrated flux densities of our VLA $6 \mathrm{~cm}$ sources within the Effelsberg beam $\left(2.6^{\prime}\right)$.

For one of the single dish sources (44.786-0.490) the peak is outside our survey area. We do not detect this source at either 6 or $3.6 \mathrm{~cm}$, probably because it is resolved out in our interferometric observations. The last two sources in Table 4 are in the region covered only at $3.6 \mathrm{~cm}$, they have been detected at this wavelength and correspond to sources $[18+19+33]$ and 32 in Table 1 . The other four sources have been detected in our $6 \mathrm{~cm}$ observations, and our integrated flux densities are in reasonable agreement with the single dish ones, except for 45.341-0.370 (our source 26) which is known to be a highly variable source (see e.g. Harmon et al. 1997). Somewhat surprisingly, in our VLA $6 \mathrm{~cm}$ images we recover almost all the single dish flux for the extended complexes $45.066-0.135$ and $45.125+0.136$, while about half of the single dish flux is recovered for $45.202-0.411$.

\subsection{IRAS Point Sources Catalogue}

To search for far infrared (FIR) counterparts to our detected radio sources, we extracted from the IRAS-PSC2 (Beichman et al. 1988) catalogue all the sources inside our survey area. In Fig. 4c we show the positions of all (43) IRAS point sources inside the observed field. We could find an IRAS counterpart within $100^{\prime \prime}$ only for 5 of our 3.6 and/or $6 \mathrm{~cm}$ sources. In all five cases, the IRAS error ellipse contains the radio continuum source or overlaps with the boundaries of the extended radio sources. In fact, in all five cases the distance from the peak of the radio continuum source and the nominal IRAS position is less than $30^{\prime \prime}$. The FIR fluxes of these five sources are reported in Table 3, Cols. 7 to 10 .

The study of the IRAS color-color diagram is a powerful tool to investigate the nature of the FIR sources. Different types of objects tend to populate different parts 
Table 3. Radio continuum spectral indexes and IRAS fluxes for the detected sources

\begin{tabular}{|c|c|c|c|c|c|c|c|c|c|c|}
\hline$\#$ & $\alpha_{3.6-6}$ & $\alpha_{6-\mathrm{ZGS}}$ & $\alpha_{3.6-\mathrm{ZGS}}$ & $\alpha_{6-\mathrm{NVSS}}$ & $\alpha_{3.6-\mathrm{NVSS}}$ & $\begin{array}{r}F_{12 \mu \mathrm{m}} \\
(\mathrm{Jy})\end{array}$ & $\begin{array}{r}F_{25 \mu \mathrm{m}} \\
(\mathrm{Jy})\end{array}$ & $\begin{array}{r}F_{60 \mu \mathrm{m}} \\
(\mathrm{Jy})\end{array}$ & $\begin{array}{r}F_{100 \mu \mathrm{m}} \\
(\mathrm{Jy})\end{array}$ & ID \\
\hline 01 & $-1.29 \pm 0.90$ & - & - & - & - & & & & & \\
\hline 02 & $-0.13 \pm 1.10$ & - & - & - & - & & & & & Cand HII \\
\hline 03 & $>+1.01$ & - & - & - & - & & & & & Cand HII \\
\hline 04 & $<-1.50$ & - & - & - & - & & & & & \\
\hline 05 & $-0.73 \pm 0.62$ & - & - & $+0.10 \pm 0.19$ & $-0.16 \pm 0.17$ & & & & & \\
\hline 06 & $-1.07 \pm 1.38$ & - & - & - & - & & & & & \\
\hline 07 & $<-0.06$ & - & - & - & - & & & & & \\
\hline 08 & $+0.93 \pm 0.90$ & - & - & - & - & & & & & Cand HII \\
\hline 09 & $-0.18 \pm 0.16$ & - & - & $+1.45 \pm 0.12$ & $+0.95 \pm 0.07$ & & & & & Cand HII \\
\hline 10 & $-0.77 \pm 0.76$ & - & - & - & - & & & & & \\
\hline $11^{a}$ & $+0.86 \pm 0.30^{a}$ & - & - & - & - & & & & & Cand HII \\
\hline $12^{a}$ & $+0.86 \pm 0.30^{a}$ & - & - & - & - & & & & & Cand HII \\
\hline 13 & $-^{b}$ & - & - & - & - & & & & & \\
\hline 14 & $-^{b}$ & $-0.31 \pm 0.33$ & $-^{b}$ & $-0.43 \pm 0.31$ & $-^{b}$ & & & & & \\
\hline 15 & $+0.23 \pm 0.04$ & $+0.59 \pm 0.09$ & $+0.47 \pm 0.07$ & $+0.51 \pm 0.08$ & $+0.42 \pm 0.06$ & 250 & 1400 & 5900 & 7500 & HII \\
\hline 16 & $-^{b}$ & - & - & - & - & & & & & \\
\hline 17 & $-0.29 \pm 0.45$ & $-0.02 \pm 0.19$ & $-0.11 \pm 0.13$ & - & - & & & & & Cand HII \\
\hline $18^{c}$ & - & - & - & - & - & & & & & $\mathrm{HII}^{c}$ \\
\hline 19 & - & - & $+0.94 \pm 0.11$ & - & - & & & & & Cand HII \\
\hline 20 & $<-0.46$ & - & - & - & - & & & & & \\
\hline 21 & $<-1.21$ & - & - & - & - & & & & & \\
\hline 22 & $-0.06 \pm 0.67$ & - & - & - & - & & & & & Cand HII \\
\hline 23 & $<-2.33$ & - & - & - & - & & & & & \\
\hline 24 & $<-0.62$ & - & - & - & - & & & & & \\
\hline 25 & $-0.94 \pm 0.23$ & $-0.90 \pm 0.11$ & $-0.91 \pm 0.12$ & $-0.77 \pm 0.10$ & $-0.83 \pm 0.11$ & & & & & \\
\hline 26 & $<-2.38$ & - & - & - & - & & & & & \\
\hline 27 & - & $-1.03 \pm 0.13$ & - & $-0.57 \pm 0.12$ & - & & & & & \\
\hline 28 & - & - & - & - & - & & & & & \\
\hline 29 & - & $-1.09 \pm 0.17$ & - & - & - & & & & & Variable? \\
\hline 30 & $+0.39 \pm 0.36$ & - & - & $-0.11 \pm 0.16$ & $+0.04 \pm 0.11$ & 58 & 490 & $<5900$ & $<7500$ & HII \\
\hline 31 & $-0.26 \pm 0.36$ & $-0.10 \pm 0.17$ & $-0.15 \pm 0.12$ & $-0.16 \pm 0.16$ & $-0.19 \pm 0.11$ & & & & & HII \\
\hline 32 & - & - & $-0.13 \pm 0.12$ & - & $-0.06 \pm 0.11$ & 37 & 303 & 2600 & $<7900$ & HII \\
\hline $33^{c}$ & - & - & $+0.42 \pm 0.10$ & - & $-0.03 \pm 0.10$ & 79 & 640 & 5300 & 7900 & $\mathrm{HII}^{c}$ \\
\hline 34 & $-0.57 \pm 0.37$ & - & - & $+0.51 \pm 0.16$ & $+0.18 \pm 0.11$ & 6.9 & 34 & 280 & 490 & HII \\
\hline
\end{tabular}

${ }^{a}$ ) Sources \#11 and \#12 are blended together at $6 \mathrm{~cm}$, the separation of the two contribution to the integrated flux is very uncertain, thus we calculated the spectral index using the integrated flux of both components together.

$\left.{ }^{b}\right)$ For these sources, due to the confusion and noise in the $8.4 \mathrm{GHz}$ map it is difficult to obtain a reliable estimate of the upper limit on the spectral index.

${ }^{c}$ ) Source \#18 is inside the extended source \#33. The total integrated flux density (4.5 Jy) has been used to determine the spectral indexes (reported only for \#33).

of the color-color planes. In Fig. 7 we show three of the color-color diagrams that can be constructed using the four IRAS fluxes, and that have been shown to be able to separate different types of galactic sources (e.g. Eder et al. 1988; Pottasch et al. 1988; WC89 White, Becker \& Helfand 1991). In each diagram the contour plots represent the normalized surface density of the colors $\left(\left[\lambda_{i}, \lambda_{j}\right] \equiv\right.$ $\left.\log _{10}\left(F_{\lambda_{i}} / F_{\lambda_{j}}\right)\right)$ of IRAS-PSC2 sources within the inner galactic plane, defined as: $|l| \leq 90^{\circ},|b| \leq 0$.65.

We note that the 43 IRAS sources in our field tend to populate the color-color planes in the same fashion as the entire inner galactic plane sample (contour plots), which, of course, is what we expected. It is remarkable, however, that all, and only, the IRAS sources detected in radio continuum (marked with plus symbols in the figure) lie in well-defined, low-density parts of the planes. This is the part of the color-color planes where ultra compact HII (UCHII) regions are expected to be found (WC89; Wood \& Churchwell 1989b; Kurtz, Churchwell \& Wood 1994; White et al. 1991; Becker et al. 1994).

\section{Discussion}

\subsection{Classification of radio sources}

We shall now classify the sources detected in our survey and reported in Table 1 according to morphology, spectral index and coincidence with IRAS sources. 

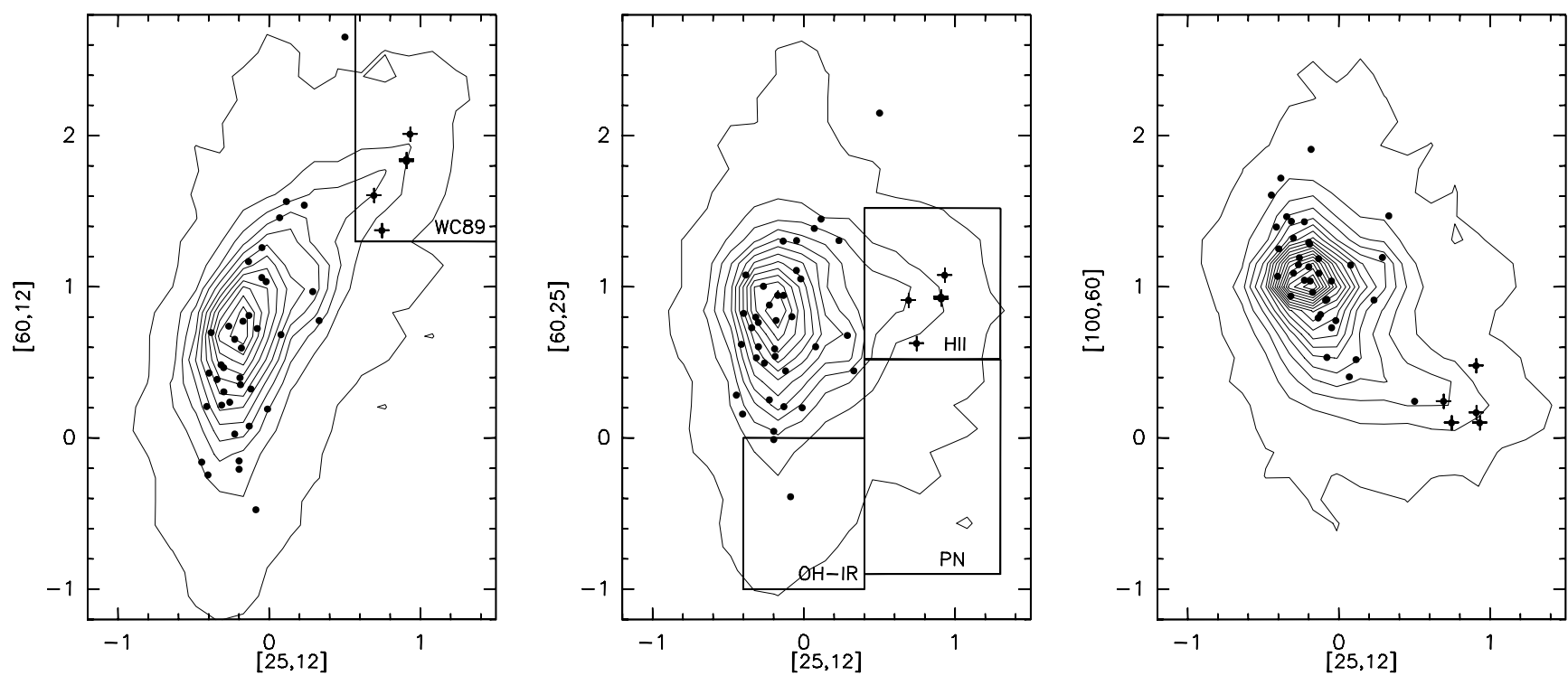

Fig. 7. In each diagram: $\left[\lambda_{i}, \lambda_{j}\right] \equiv \log _{10}\left(F_{\lambda_{i}} / F_{\lambda_{j}}\right)$; the contour plots represent the normalized surface density of IRAS-PSC2 sources in the region $|l| \leq 90^{\circ},|b| \leq 0^{\circ} 65$. Black filled circles show the colors of the 43 sources in our surveyed region (one source detected only at $100 \mu \mathrm{m}$ is not present in the first two plots). Many of the sources only have upper limits at one or more IRAS bands, the colors for these sources are either upper, lower limits, or are undetermined. We have not marked these sources with special symbols as we have not corrected the color-color surface density contours for upper limits. The five IRAS sources with a radio continuum counterpart are marked with plus symbols

Table 4. Comparison between single dish and VLA $5 \mathrm{GHz}$ sources

\begin{tabular}{crrl}
\hline Effelsberg & \multicolumn{2}{l}{ VLA } \\
Name & $\begin{array}{r}F_{\mathrm{p}} \\
(\mathrm{Jy})\end{array}$ & $\begin{array}{r}F_{\mathrm{i}} \\
(\mathrm{Jy})\end{array}$ & $\begin{array}{l}\text { Sources ID from } \\
\text { Table 1 }\end{array}$ \\
\hline $44.786-0.490$ & 0.2 & - & Not detected, high rms \\
$45.066-0.135$ & 0.7 & 0.62 & 11,12, and 30 \\
$45.125+0.136$ & 5.8 & 5.25 & $13-17$, and 31 \\
$45.202-0.411$ & 0.2 & 0.096 & 34 \\
$45.341-0.370$ & 0.2 & 0.002 & $26^{a}$ \\
$45.451+0.060$ & 6.4 & - & Not mapped at $6 \mathrm{~cm}$ \\
$45.475+0.130$ & 2.1 & - & Not mapped at $6 \mathrm{~cm}$ \\
\hline
\end{tabular}

a) This source is known to be variable (e.g. Harmon et al. 1997).

Five complexes of sources have all the specifications for being classified as thermal galactic HII regions. They include all the extended sources plus some additional small diameter source in the same area, more precisely $[11+12$ $+30],[13+15+16+17+31],[34],[18+33]$ and [32] (numbers are from Table 1). All these complexes coincide with corresponding sources in the Altenhoff et al. (1978) survey (see Sect. 3.2) and are now resolved in much more detail. Morphologically they show the classical aspect of a cluster of HII regions, of which G9.62+0.19 is a typical example (Testi et al. 1998, 1999), i.e. several sources of different angular sizes (from unresolved to several tens of $\operatorname{arcsec}$ ) are clustered in the same area. The continuum sources may represent independent UCHII regions born in the same star forming complex but presently observed in different evolutionary phases with the unresolved sources being the youngest and the more extended sources more evolved.

Six of the small diameter sources $(2,3,8,9,19,22)$ can be classified as "candidate HII region" according to their spectral index. No IRAS source is associated with them, but their radio flux is rather low. Non detection at far infrared wavelengths could be either due to the intrinsic weakness of some of these sources or, most probably, due to the incompleteness of the IRAS-PSC in the galactic plane (see also Becker et al. 1994).

The remaining 15 sources $(4,5,6,7,10,14,20,21,23$, $24,25,26,27,28$ and 29) can be classified from the spectral index as non thermal (probably extragalactic) sources. Only five of these have been detected at $20 \mathrm{~cm}$. These have in general greater integrated flux densities at $6 \mathrm{~cm}$ than those not detected at $20 \mathrm{~cm}$ (the mean $6 \mathrm{~cm}$ flux densities of the two groups are 10 and $2 \mathrm{mJy}$, respectively), so that the effect can be simply explained as due to our higher sensitivity at $6 \mathrm{~cm}$. All 15 sources have been detected at $6 \mathrm{~cm}$ and 4 of them at $3.6 \mathrm{~cm}$ as well. Given the area observed at $6 \mathrm{~cm}(0.620 \mathrm{sq}$. deg.) and that observed at $3.6 \mathrm{~cm}$ (0.525 sq. deg.), the number of extragalactic sources above the $1 \mathrm{mJy}$ threshold, which we can assume as a mean detection limit for our survey, can be estimated from deep VLA surveys. Following Fomalont et al. (1991) at $6 \mathrm{~cm}$ we expect 15 extragalactic sources above our $1 \mathrm{mJy}$ threshold, while at $3.6 \mathrm{~cm}$ the number is reduced to 9 sources for the same threshold (Windhorst et al. 1993). Given the 
small number statistics, these numbers are in relatively good agreement with the source counts in our surveyed area.

Becker et al. (1994) estimated a total of $\sim 100$ planetary nebulae $(\mathrm{PNs})$ down to a flux limit of $\sim 2.5 \mathrm{mJy}$ in their $6 \mathrm{~cm}$ survey of $50 \mathrm{sq}$. deg. of the inner galactic plane. This number correspond to less than 2 PNs expected down to the same flux level in our $6 \mathrm{~cm}$ survey region. Thus the contamination from PNs in our source lists should be very small.

\subsection{IRAS "UCHII-type" sources}

In Sect. 3.4 it was pointed out that all the IRAS sources with a corresponding radio counterpart in our survey $(5$ out of 43) satisfy the color-color criteria to be classified as UCHII regions (WC89). However, with the possible exception of the double source G045.070+0.132 and G045.072+0.132 (11 and 12), none of the radio sources within $100^{\prime \prime}$ from the IRAS-PSC position can be classified as bona fide UCHII region using the usual definition (Wood \& Churchwell 1989b; Kurtz et al. 1999a). The radio continuum sources are extended (non homogeneous) HII regions, with emission peaks inside them that may appear as UCHII regions when observerved with an extended VLA configuration. A typical example could be G045.455+0.060 which appears as a compact source inside the extended HII region G045.455+0.059 (see Fig. A5), this source has the appearence of an UCHII region in the Wood \& Churchwell (1989b) survey (their source G45.45+0.06). The VLA high frequency and high resolution surveys of IRAS selected UCHII candidates are all biased to the detection of only the most compact and dense ionized gas, due to the spatial filtering of the interferometer, and are unable to detect the extended components. Our results agree with those of Kurtz et al. (1999b) and show that, when observed with sufficient sensitivity to extended structures, most, if not all, the IRAS selected UCHII candidates do have extended radio components. This implies that samples of IRAS-PSC sources selected with the WC89 criteria are contaminated by a substantial number of older more extended HII regions (see also Codella et al. 1994; Ramesh \& Sridharan 1997; Kurtz et al. 1999b). The number of UCHII regions estimated from the color selected IRAS-PSC counts may be, consequently, overestimated by a large factor. If most IRAS-WC89 sources are indeed associated with extended HII rather than UCHII regions, the lifetime of the IRAS-WC89 color phase of UCHII/HII regions may be much longer than estimated from the early high resolution radio surveys. Consequently, the estimated lifetime of the UCHII phase for O-type young stars is probably much shorter that previously thought (see also Ramesh \& Sridharan 1997). Additionally, we find 6 UCHII candidates in our radio continuum survey without an associated
IRAS source. As discussed by Becker et al. (1994), this is probably due to the confusion limit of the PSC on the galactic plane, and the generally lower radio luminosity of these sources. However, we note that in our field only unresolved thermal radio sources are not present in the IRAS-PSC, while all resolved HII regions are detected in the far-infrared. Incidentally, we note that all the compact thermal radio sources in our survey not associated with IRAS PSC sources are fainter at centimeter wavelengths than those detected in the far infrared, and thus they may be associated with stars of type later than $\mathrm{O}$. However, without knowing the distances it is impossible to draw a final conclusion.

In our surveyed region, the percentage of IRAS sources satisfying WC89 color criteria is $(5 / 43 \sim 12 \%)$. This is consistent with the percentage found accross the entire inner galactic plane $\left(|l| \leq 90^{\circ},|b| \leq 0 \% 6, \sim 8 \%\right)$. The fraction of WC89 sources in the IRAS-PSC database drops to much lower values outside the inner galactic plane (WC89).

\subsection{Continuum emission from the $\mathrm{H}_{2} \mathrm{O}$ maser}

During an incomplete low spatial resolution $\left(2^{\prime}\right)$ single dish survey of the $l=+45^{\circ}$ field in the $\mathrm{H}_{2} \mathrm{O} 22 \mathrm{GHz}$ maser line, a new maser was detected. The masing gas is probably coincident with a $15 \mu \mathrm{m}$ source $\left(F_{15} \mu \mathrm{m}=370 \mathrm{mJy}\right)$ located at $\alpha(2000)=19^{\mathrm{h}} 12^{\mathrm{m}} 46^{\mathrm{s}} \delta(2000)=10^{\circ} 45^{\prime} 30^{\prime \prime}$, and was interpreted as a candidate young stellar object (Testi et al. 1997). Therefore, it was interesting to see if any radio continuum emission from an associated UC HII region could be detected.

From a careful inspection of the area around the maser, no radio continuum emission was seen above the (local) $3 \sigma$ level $(0.6 \mathrm{mJy} /$ beam at $3.6 \mathrm{~cm}$ and $1.2 \mathrm{mJy} /$ beam at $6 \mathrm{~cm})$. With the young stellar object hypothesis in mind, there are two possible explanations: 1) the putative UCHII region is intrinsically too weak to be detected or absent because the eventual exciting star is of late spectral type; or 2) there is an UCHII region, but it is in such an early evolutionary phase that it is optically thick even at $3.6 \mathrm{~cm}$. The lack of radio continuum emission close to $\mathrm{H}_{2} \mathrm{O}$ masers in high luminosity star forming regions has been amply demonstrated by a survey of a large number of maser in the radio continuum, which showed that many maser associated with high luminosity sources do not have any close-by radio continuum source (Tofani et al. 1995). Subsequent molecular observations of the masers without continuum emission has indeed confirmed that these are associated with very young star forming regions since in all cases a hot molecular core was found at the same position (Cesaroni et al. 1999).

To settle the nature of the new maser-15 $\mu \mathrm{m}$ source, molecular observations in high density tracers are needed, as well as an estimate of its distance and luminosity. 
$\mathrm{G} 044.841+0.554$

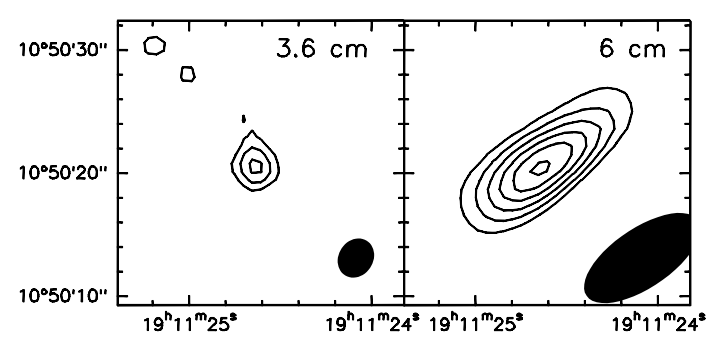

G044.854+0.519

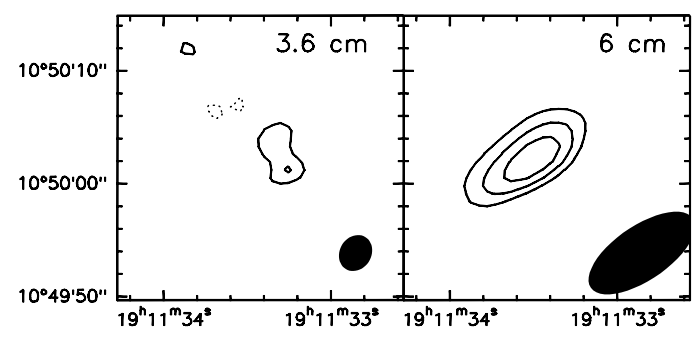

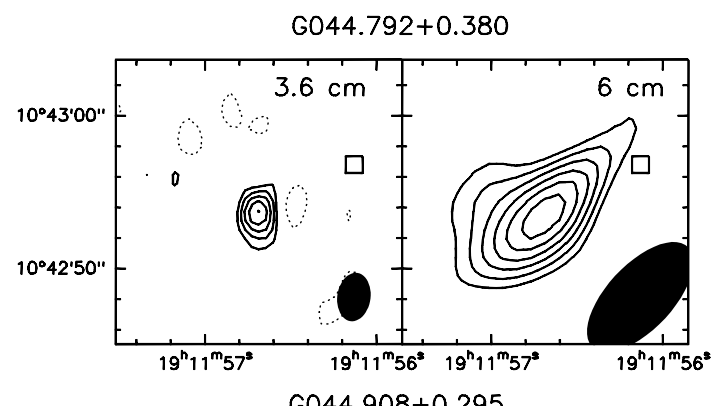

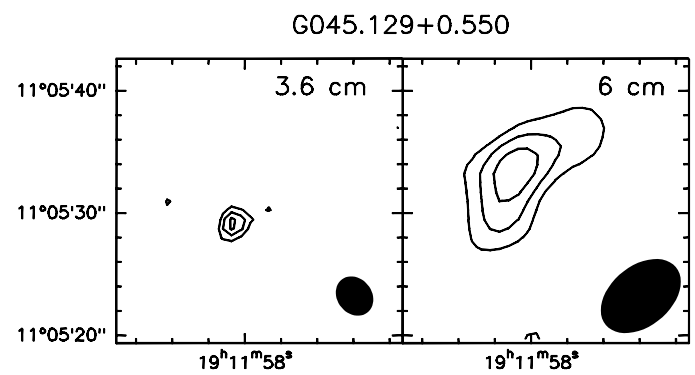

$\mathrm{G} 044.965+0.284$
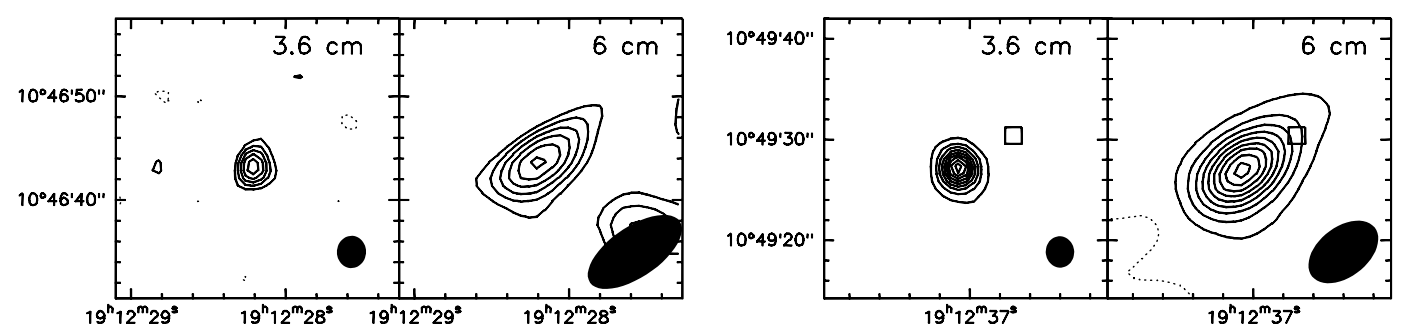

$\mathrm{G} 045.027+0.123$
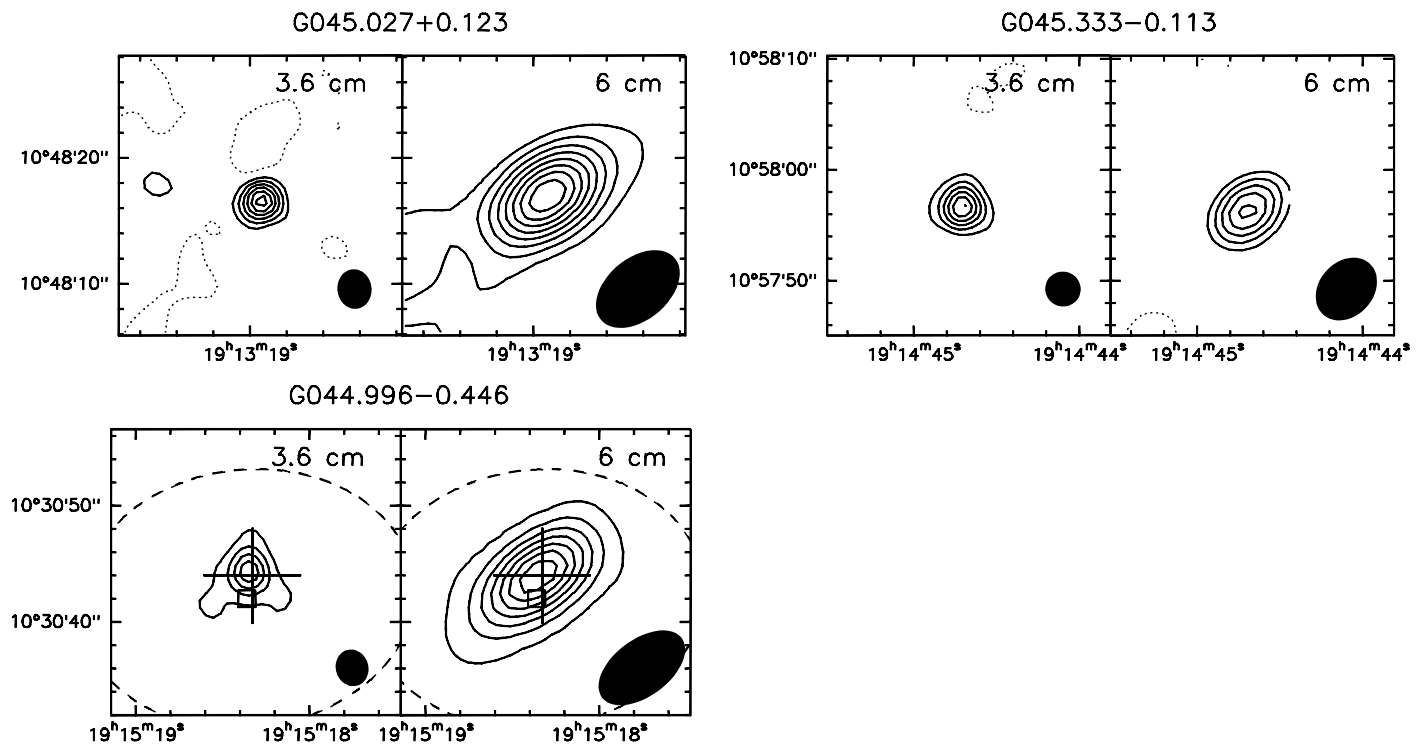

Fig. A1. 3.6 and $6 \mathrm{~cm}$ maps of the unresolved sources detected at both frequencies; crosses mark the peak positions of $20 \mathrm{~cm}$ sources from Zoonematkermani et al. (1990); open sqares mark the positions of the peaks of NVSS sources (Condon et al. 1998), the deconvolved FWHM of these sources are shown as dashed ellipses, which may be partially outside the plotted area 

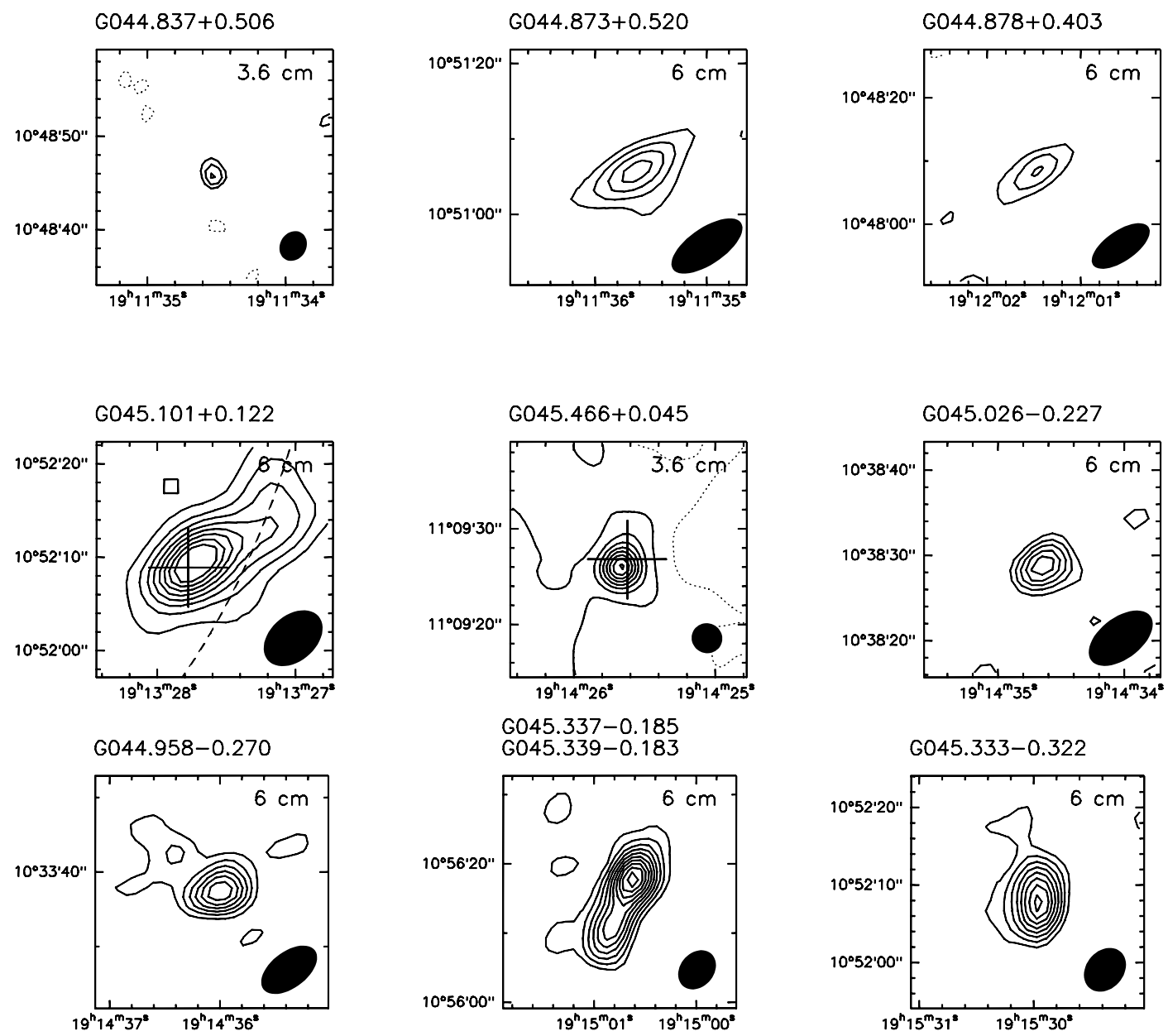

\section{G045.337-0.185}

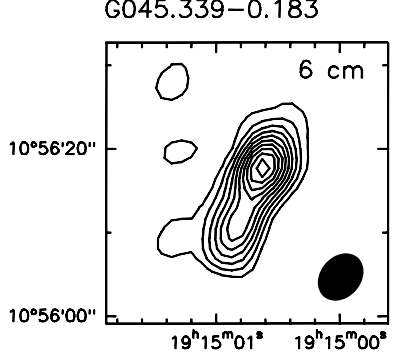

G045.333-0.322
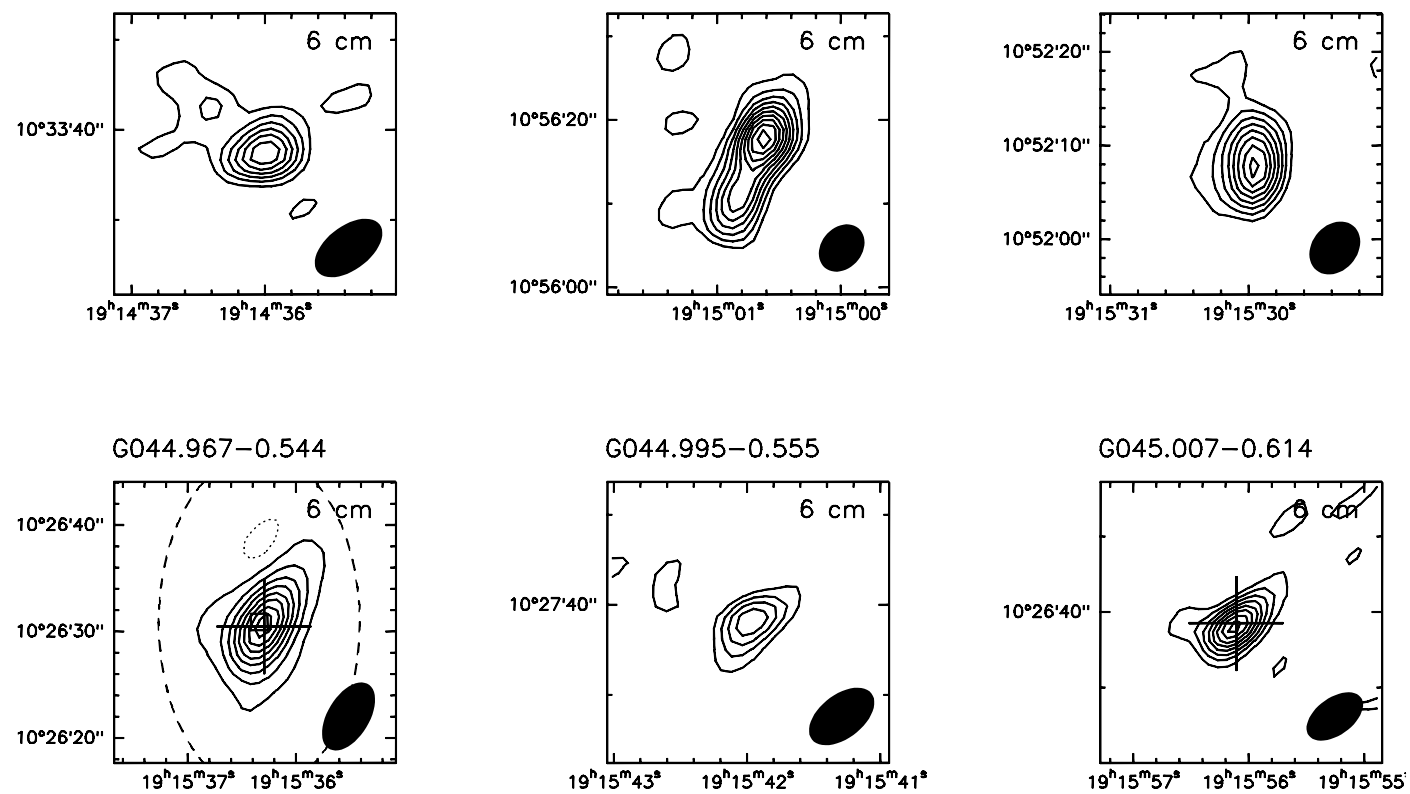

Fig. A2. As Fig. A1 but for sources detected only at one frequency

\section{Conclusions}

The unbiased radio continuum survey of the ISOGAL field at $l=+45^{\circ}$ has resolved the structure of five thermal extended complexes and discovered 21 additional small diameter sources, six of which are candidate HII regions.

Comparison with the IRAS PSC shows that all 5 of the extended thermal sources have corresponding FIR emission and that the colors of these sources satisfy the WC89 color criteria for UCHII. Our sources, however, are not UCHII regions, but are more evolved extended HII regions. This result is consistent with the results of earlier single dish surveys (Codella et al. 1994) and of a recent survey for extended emission around IRAS-selected UCHII regions (Kurtz et al. 1999b).

We conclude that UCHII counts based on IRAS selected samples are overestimated by a large factor, consequently the estimated lifetime of the UCHII phase may be substantially reduced, removing the so-called lifetime problem for UCHII regions.

The percentage of IRAS sources associated with HII regions is $\sim 10 \%$ in our field, which seems to be a general property of IRAS sources in the galactic plane. 


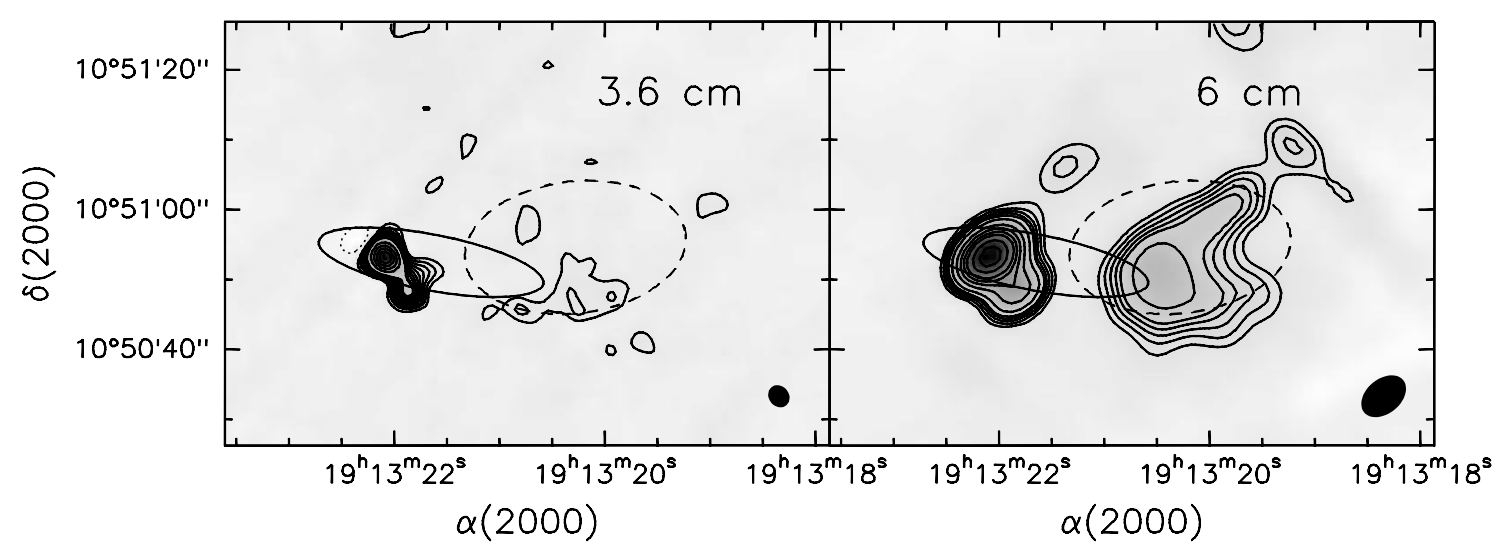

Fig. A3. 3.6 and $6 \mathrm{~cm}$ maps of the extended source G045.066+0.138 (30), and the compact sources G045.070+0.132 (11) and G045.072+0.132 (12); contour levels are -8 , from 8 to 50 by 5 and from 50 to 300 by $50 \mathrm{mJy} /$ beam at $3.6 \mathrm{~cm}$, and -10 , from 10 to 22 by 3 , from 30 to 90 by 15, 120 and $150 \mathrm{mJy} /$ beam at $6 \mathrm{~cm}$. The filled ellipse represent the IRAS-PSC error box

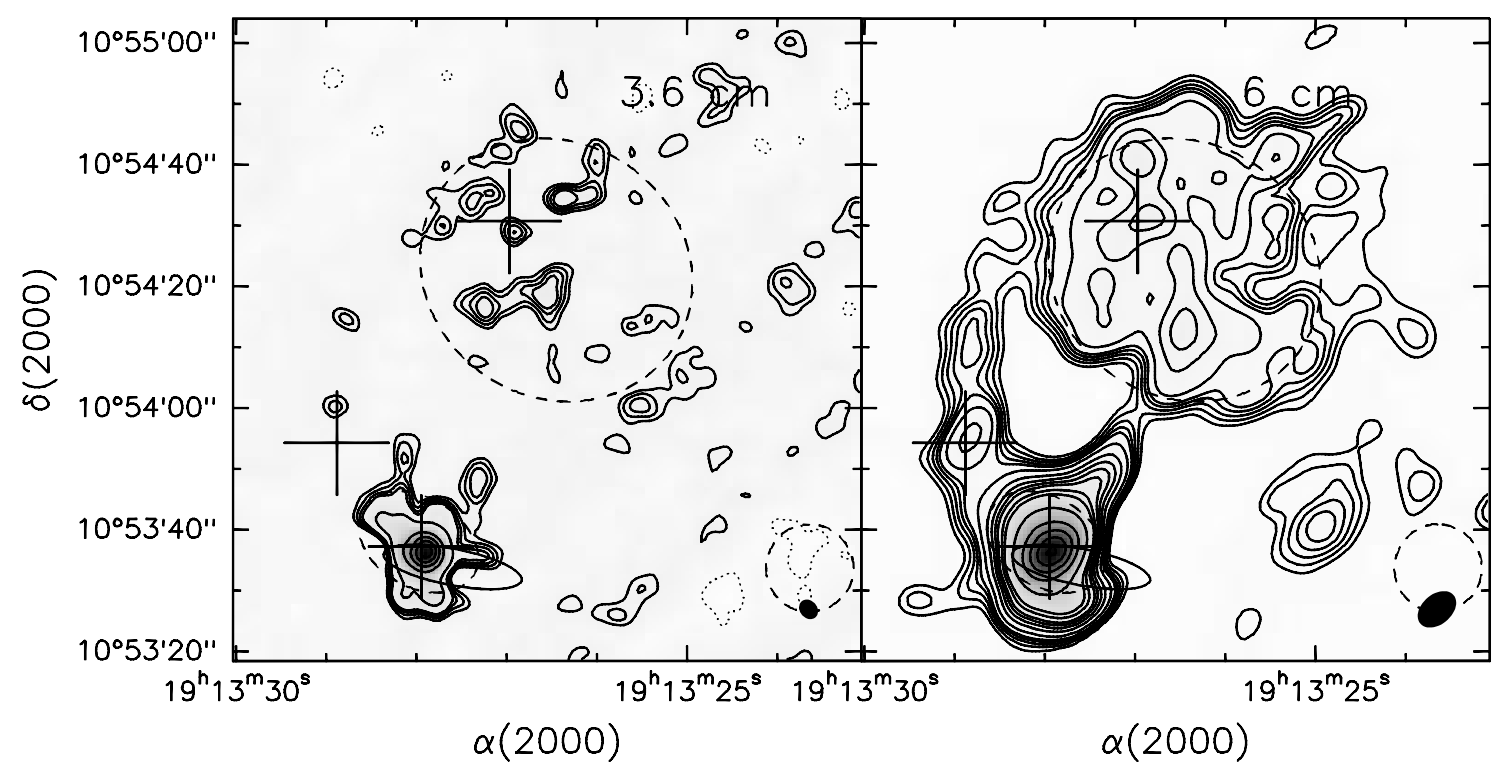

Fig. A4. 3.6 and $6 \mathrm{~cm}$ maps of the extended source G045.134+0.145 (31), and the compact sources G045.118+0.143 (13), G045.123+0.132 (15), G045.133+0.133 (16) and G045.130+0.131 (17); contour levels are -30, from 30 to 100 by 10 and from 200 to 1400 by $300 \mathrm{mJy} /$ beam at $3.6 \mathrm{~cm}$, and -10 , from 10 to 22 by 3 , from 30 to 90 by 15, 120, 150 and from 300 to 1500 by $300 \mathrm{mJy} /$ beam at $6 \mathrm{~cm}$. The filled ellipse represent the IRAS-PSC error box

Acknowledgements. Support from CNR-NATO Advanced Fellowship program and from NASA's Origins of Solar Systems program (through grant NAGW-4030) is gratefully acknowledged.

\section{Appendix A: Maps of all the detected radio sources}

In this appendix we present the contour plots of all the sources detected in our survey. IRAS-PSC2 sources are shown as ellipses corresponding to the $90 \%$ confidence on the peak position. The positions of the peaks of the $20 \mathrm{~cm}$ sources from Zoonematkermani et al. (1990) are shown as crosses. Dashed ellipses show the deconvolved sizes of the $20 \mathrm{~cm}$ NVSS survey sources (Condon et al. 1998). In Figs. A1 and A2 the NVSS ellipses may fall partially (or completely) outside the plotted area, thus we marked the NVSS peak position with an empty square.

\section{References}

Altenhoff W.J., Downes D., Pauls T., Schraml J., 1978, A\&AS 35,23

Becker R.H., White R.L., Helfand D.J., Zoonematkermani S., 1994, ApJS 91, 347 

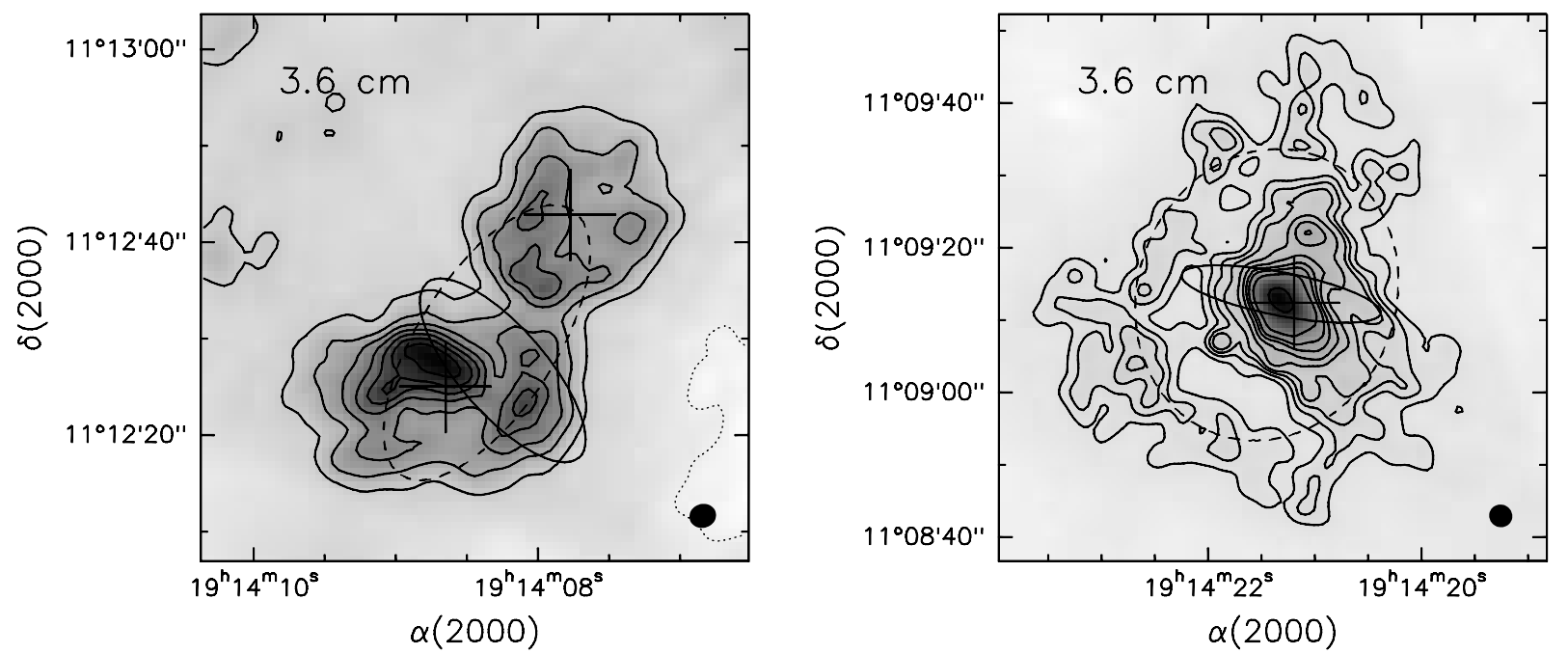

Fig. A5. $3.6 \mathrm{~cm}$ maps of the extended sources G045.479+0.130 (32) on the left panel and 045.455+0.059 (33) and compact source G045.455+0.060 (18) on the right panel; contour levels are: -3 , from 3 to 37 by $5 \mathrm{mJy} / \mathrm{beam}$, and -9 , from 9 to 27 by 6 , from 39 to 75 by $12,100,150$ and $200 \mathrm{mJy} /$ beam. The filled ellipse represent the IRAS-PSC error box

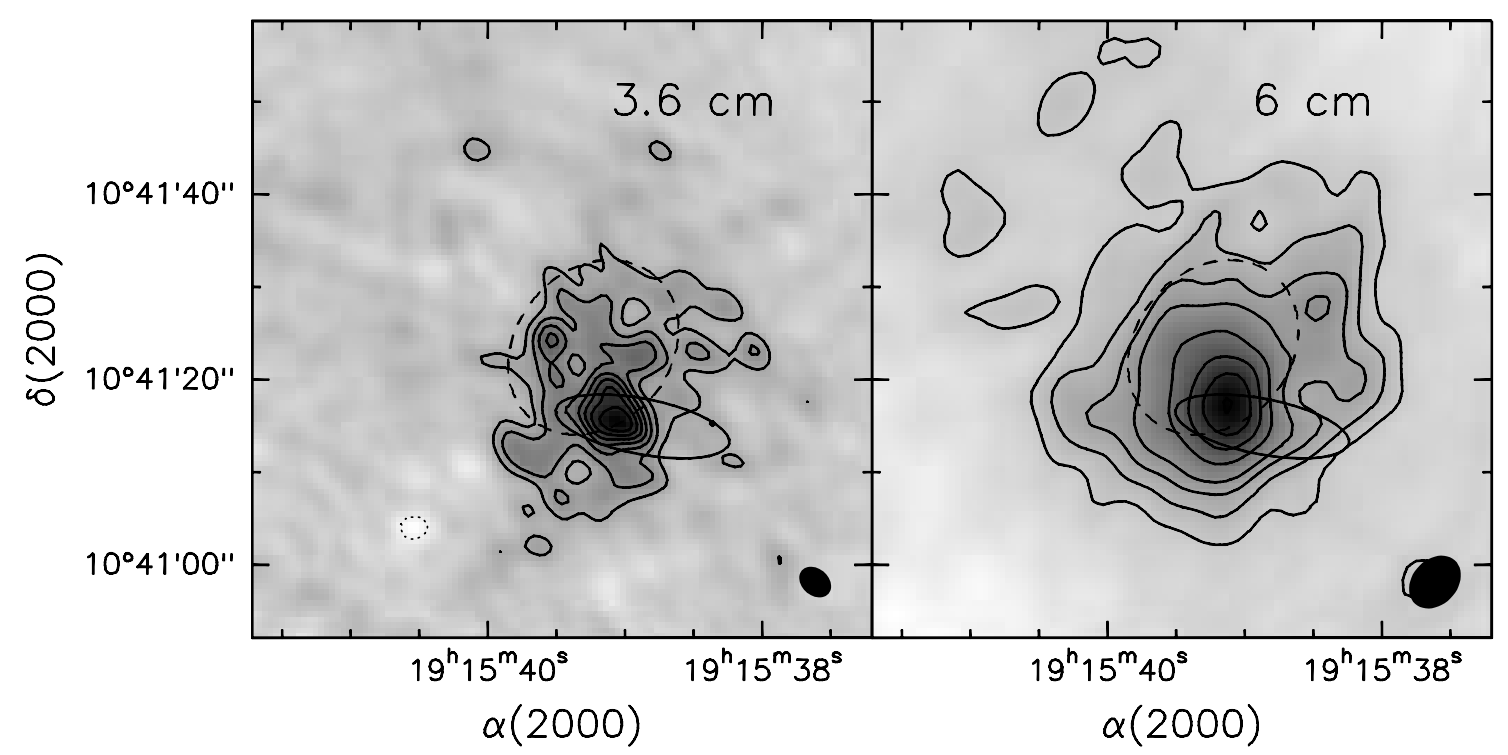

Fig. A6. 3.6 and $6 \mathrm{~cm}$ maps of the extended source G045.190-0.439 (34); contour levels are -0.6 , from 0.6 to 3.3 by $0.4 \mathrm{mJy} / \mathrm{beam}$ at $3.6 \mathrm{~cm}$, and -0.9 , from 0.9 to 2.7 by 0.6 , from 3.9 to 7.5 by $1.2 \mathrm{mJy} /$ beam at $6 \mathrm{~cm}$. The filled ellipse represent the IRAS-PSC error box

Beichman C.A., Neugebauer G., Habing H.J., Clegg P.E., Chester T.J., 1988, IRAS Catalogs and Atlases. GPO, Washington, DC

Cesaroni R., Felli M., Walmsley C.M., 1999, A\&AS 136, 333

Codella C., Felli M., Natale V., 1994, A\&A 284, 233

Condon J.J., Cotton W.D., Greisen E.W., et al., 1998, ApJ 115,1693

Eder J., Lewis B.M., Terzian Y., 1988, ApJS 66, 183

Epchtein N., 1998, IAU Symp. 191, p. 102

Fomalont E.B., Windhorst R.A., Kristian J.A., Kellerman K.I., 1991, AJ 102, 1258
Harmon B.A., Deal K.J., Paciesas W.S., et al., 1997, ApJ 477, L85

Kurtz S., Churchwell E., Wood D.O.S., 1994, ApJS 91, 659

Kurtz S., Cesaroni R., Hofner P., Churchwell E., Walmsley C.M., 1999a, in "Protostars \& Planets IV", Mannings V., Boss A., Russel S. (eds.). the Arizona University press (in press)

Kurtz S., Watson A.M., Hofner P., Otte B., 1999b, ApJ 514, 232

Mirabel I.F., Rodríguez L.F., 1994, Nat 371, 46

Omont A., Blommaert J., 1997, in "The impact of large-scale Near-IR Sky Surveys", Garzón P. et al. (ed.). Kluwer, p. 81 
Pérault M., Omont A., Simon G., Séguin P., Ojha D., et al., 1996, A\&A 315, L165

Pottasch S.R., Bignell C., Olling R., Zijlstra A.A., 1988, A\&A 205, 248

Ramesh B., Sridharan T.K., 1997, MNRAS 284, 1001

Rodríguez L.F., Mirabel I.F., 1999, ApJ 511, 398

Testi L., Felli M., Pérault M., et al., 1997, A\&A 318, L13

Testi L., Felli M., Persi P., Roth M., 1998, A\&A 329, 233

Testi L., Hofner P., Kurtz S., Rupen M., Cesaroni R., 1999 (in preparation)
Tofani G., Felli M., Taylor G.B., Hunter T.R., 1995, A\&AS 112,299

White R.L., Becker R.H., Helfand D.J., 1991, ApJ 371, 148

Windhorst R.A., Fomalont E.B., Partridge R.B., Lowenthal J.D., 1993, ApJ 405, 498

Wood D.O.S., Churchwell E., 1989a, ApJ 340, 265

Wood D.O.S., Churchwell E., 1989b, ApJS 69, 831

Zoonematkermani S., Helfand D.J., Becker R.H., White R.L., Perley R.A., 1990, ApJS 74, 181 